Lorenzo Mazzieri

\title{
Generalized connected sum construction for scalar flat metrics
}

Received: 10 December 2007 / Accepted: 13 December 2008

Published online: 10 February 2009

(C) The Author(s) 2009. This article is published with open access at Springerlink.com

\begin{abstract}
In this paper we construct constant scalar curvature metrics on the generalized connected sum $M=M_{1} \sharp_{K} M_{2}$ of two compact Riemannian scalar flat manifolds $\left(M_{1}, g_{1}\right)$ and $\left(M_{2}, g_{2}\right)$ along a common Riemannian submanifold $\left(K, g_{K}\right)$ whose codimension is $\geq 3$. Here we present two constructions: the first one produces a family of "small" (in general nonzero) constant scalar curvature metrics on the generalized connected sum of $M_{1}$ and $M_{2}$. It yields an extension of Joyce's result for point-wise connected sums in the spirit of our previous issues for nonzero constant scalar curvature metrics. When the initial manifolds are not Ricci flat, and in particular they belong to the $\left(1_{+}\right)$class in the Kazdan-Warner classification, we refine the first construction in order to produce a family of scalar flat metrics on $M$. As a consequence we get new solutions to the Einstein constraint equations on the generalized connected sum of two compact time symmetric initial data sets, extending the Isenberg-Mazzeo-Pollack gluing construction.
\end{abstract}

\section{Introduction and statement of the results}

This last two decades gluing techniques for solutions of nonlinear problems have been successfully applied in several situations. They have been used to understand solutions to problems arising from the geometry (minimal and constant mean curvature surfaces $[13,14]$, constant scalar curvature metrics $[9,12,15]$, and recently even Einstein metrics [1]) and from the physic (Einstein constraint equations [7] and [8]). However most of the results are concerned with the connected sum at points (point-wise connected sum), whereas the case of connected sum along a submanifold (generalized connected sum or fiber sum) has received less attention. This kind of construction is clearly less flexible than the first one, nevertheless it has revealed to be a very powerful tool in studying for example the structure of the manifolds with positive scalar curvature (see $[6,17]$ ).

In this paper we will show how the generalized connected sum construction for nonzero constant scalar curvature metrics introduced in [16] can be extended to the

L. Mazzieri: Scuola Normale Superiore, Pisa, Italy

L. Mazzieri: Université Paris 12, Paris, France

Present address:

L. Mazzieri $(\varangle)$ : Max Planck Institut für Gravitationsphysik, Albert Einstein Institut, Am Mühlenberg 1, 14476 Golm, Germany. e-mail: lorenzo.mazzieri@ aei.mpg.de

Mathematics Subject Classification (2000): 53C21, 58J60, 53A30, 57R65, 83C05

DOI: $10.1007 / \mathrm{s} 00229-009-0250-\mathrm{y}$ 
case where the initial manifolds carry scalar flat metrics. In other words we produce a family of new solutions to the Yamabe equation by gluing together two compact scalar flat Riemannian manifolds $\left(M_{1}, g_{1}\right)$ and $\left(M_{2}, g_{2}\right)$, along a common submanifold $\left(K, g_{K}\right)$ of codimension $\geq 3$. The reason to requiring high codimension lies in the geometry of the construction. In fact in order to produce the generalized connected sum $M=M_{1} \sharp_{K} M_{2}$ we assume that the normal bundles of $K$ in $M_{1}$ and $M_{2}$ are diffeomorphic, then we remove from both the initial manifolds a tubular neighborhood of $K$ of size $\varepsilon>0$ and we perform a fiber-wise identification between the left over boundaries (fiber sum). This way we obtain a sort of polyneck which glues together the differential structures of $M_{1}$ and $M_{2}$, but we still need to identify the Riemannian structures. If $m$ is the dimension of the starting manifolds, $k$ is the dimension of $K$ and $n:=m-k$ its codimension in $M_{i}, i=1,2$, it turns out (see $[9,16])$ that a good choice is to model the metric of the polyneck fiber-wise around an $n$-dimensional Schwarzschild metric, whose existence requires that $n$ has to be $\geq 3$.

The reason why we treat the scalar flat case independently from the nonzero constant scalar curvature case (i.e. when the initial manifolds have the same constant scalar curvature $S \neq 0$ ) is that the analysis is rather different. In fact the assumption that the linearized Yamabe operators $\Delta_{g_{i}}+S /(m-1)$ of the initial metrics $g_{i}, i=1,2$ are injective, is crucial to carry out the proof in the nonzero case. In the scalar flat situation, the linearized operator reduces to the Laplacian for which this assumption is not fulfilled and this will force us to work orthogonally with respect to the kernel of the Laplacian (namely the space of constant functions). In addition, it turns out that, on the generalized connected sum, the first nonzero eigenvalue of the Laplacian is very small and actually tends to zero as $\varepsilon \rightarrow 0$. This fact will make the search of suitable a priori estimates for the linearized operator harder. Because of this, when we will perform the nonlinear analysis, we will have to take some care in estimating the projection of the error terms over the eigenfunction associated to the small eigenvalue. We will show that, if the construction is done with care, such a projection can be chosen to be zero.

We present in this paper two kinds of construction. The first one is more general than the second one but it has a major drawback. In fact following this method we are not allowed to choose a scalar flat metric on the generalized connected sum, although the error can be chosen as small as we want (notice that similar phenomena happen in the point-wise connected sum case [9]). The second construction is an improvement of the first one and enables us to obtain scalar flat metrics on the final manifold, but it requires the hypothesis that the summands are non Ricci flat. In particular, in order to obtain a scalar flat generalized connected sum, it is necessary that both the manifolds $M_{1}$ and $M_{2}$ belong to the $\left(1_{+}\right)$class in the Kazdan-Warner classification $[10,11]$. An important corollary of the second construction is that it provides a gluing construction for time symmetric initial data sets in the context of the Einstein Constraint equations. In this sense our result partially completes the work of Isenberg et al. [8], which treats the point-wise connected sum of nontime symmetric Cauchy data.

In Sects. 2-5 we present the first method. As in the non scalar flat case, we write down a family of approximate solution metrics $\left(g_{\varepsilon}\right)_{\varepsilon \in(0,1)}$ (where the parameter $\varepsilon>0$ represents the size of the tubular neighborhood we excise from each 
manifold in order to perform the generalized connected sum) and then we find out a conformal factor $u_{\varepsilon}$ such that for sufficiently small $\varepsilon>0$ the metrics $\tilde{g}_{\varepsilon}=u_{\varepsilon}^{\frac{4}{m-2}} g_{\varepsilon}$, $\varepsilon \in(0,1)$, are (small) constant scalar curvature metrics. As mentioned before, by this method it is not possible to ensure that the scalar curvature $S=S_{\tilde{g}_{\varepsilon}}$ of the final metrics is exactly zero. However we will show that the scalar curvature of the metrics we obtain are as close to zero as we want, namely $S=\mathcal{O}\left(\varepsilon^{n-2}\right)$. Let us now describe this result more precisely.

Let $\left(M_{1}, g_{1}\right)$ and $\left(M_{2}, g_{2}\right)$ be two $m$-dimensional compact Riemannian manifolds with scalar flat metric, and suppose that there exists a $k$-dimensional Riemannian manifold $\left(K, g_{K}\right)$ which is isometrically embedded in both $\left(M_{1}, g_{1}\right)$ and $\left(M_{2}, g_{2}\right), m \geq 3, n:=m-k \geq 3$. We also assume that the normal bundles of $K$ in $\left(M_{i}, g_{i}\right)$ can be diffeomorphically identified (this is necessary to perform the fiber sum, see [17]). Another natural assumption is that the starting manifolds have the same volume and in particular we assume that $\operatorname{vol}_{g_{1}}\left(M_{1}\right)=1=\operatorname{vol}_{g_{2}}\left(M_{2}\right)$. Notice that this condition turns out to be necessary also in the case of point-wise connected sum of two scalar flat metrics [9].

Let $M_{\varepsilon}=M_{1} \sharp{ }_{K, \varepsilon} M_{2}$ (briefly $M$ ) be the generalized connected sum of $\left(M_{1}, g_{1}\right)$ and $\left(M_{2}, g_{2}\right)$ along $K$ which is obtained by removing an $\varepsilon$-tubular neighborhood of $K$ from each $M_{i}$ and identifying the two left over boundaries. Our main result reads:

Theorem 1. Under the above assumptions, there exists a real number $\varepsilon_{0}>0$ such that for every $\varepsilon \in\left(0, \varepsilon_{0}\right)$ it is possible to endow $M$ with a constant scalar curvature metric $\bar{g}_{\varepsilon}$, whose scalar curvature $S_{\bar{g}_{\varepsilon}}$ is a $\mathcal{O}\left(\varepsilon^{n-2}\right)$. In addition the metric $\bar{g}_{\varepsilon}$ is conformal to the metrics $g_{i}$ away from a fixed (small) tubular neighborhood of $K$ in $M_{i}, i=1,2$ for a conformal factor $u_{\varepsilon}$ which can be chosen so that

$$
\left\|u_{\varepsilon}-1\right\|_{L^{\infty}(M)} \leq C \cdot \varepsilon^{\gamma}
$$

where $C>0$ and $\gamma \in(0,1 / 4)$. Moreover the new metrics tend to the old ones on the compact sets of $M_{i} \backslash K, i=1,2$ in the $\mathcal{C}^{2}$ topology, as $\varepsilon \rightarrow 0$.

Section 6 is devoted to the description of the second construction, which works in the non Ricci flat case. In this situation we will be able to improve the construction of the approximate solution metrics in order to obtain a scalar flat metric on the generalized connected sum. In fact, if the starting manifolds are non Ricci flat, we are allowed to create two correction terms by means of slight non conformal modifications of the initial metrics away from the gluing locus, what enable us to ensure that the error terms are orthogonal to the space generated by the constant functions and-roughly speaking - to the first nonconstant eigenfunction (the one whose eigenvalue tends to zero in the limit for $\varepsilon \rightarrow 0$ ). This is enough to carry out the analysis and to construct on the generalized connected sum a solution of the Yamabe equation with prescribed zero scalar curvature. As already mentioned, the non Ricci flat condition on a scalar flat manifold implies that such a manifold belongs to the $\left(1_{+}\right)$class in the Kazdan-Warner classification. This seems to be quite natural since these manifolds are in some sense the most flexible ones, concerning the prescription of the scalar curvature. In other words, if a manifold $M$ is 
in class $\left(1_{+}\right)$, any smooth function on it can be viewed as the scalar curvature of some Riemannian metric $[10,11]$.

The statement of our second result is the following:

Theorem 2. Let $M$ be the generalized connected sum of two Riemannian scalar flat, non Ricci flat manifolds $\left(M_{1}, g_{1}\right)$ and $\left(M_{2}, g_{2}\right)$ of dimension $m \geq 3$ along a common isometrically embedded submanifold $\left(K, g_{K}\right)$ of codimension at least 3 (recall that in order to perform the generalized connected sum it is necessary that the normal bundles of $K$ in $M_{1}$ and $M_{2}$ are diffeomorphic). Under these assumptions, there exists a real number $\varepsilon_{0}>0$ such that, for every $\varepsilon \in\left(0, \varepsilon_{0}\right)$, it is possible to endow $M$ with a scalar flat metrics $\bar{g}_{\varepsilon}$. Moreover the new metrics tend to the old ones on the compact sets of $M_{i} \backslash K, i=1,2$ in the $\mathcal{C}^{2}$ topology, as $\varepsilon \rightarrow 0$.

In Sect. 7 we will show how the Theorem 2 applies to a problem of physical interest. It is well known [4] that a solution to the hyperbolic Einstein system in general relativity can be found by evolving a suitable initial data set (or Cauchy data set). More precisely a space-like $m$-dimensional hypersurface $M$ in a $(m+1)$ dimensional Lorentzian manifold $(Z, \gamma)$ do evolve to a solution of $\operatorname{Ric}_{\gamma}=0$ if and only if the following Einstein constraint equations are satisfied

$$
\begin{array}{r}
\operatorname{div}_{g} \Pi-d\left(\operatorname{tr}_{g} \Pi\right)=0 \\
S_{g}-|\Pi|_{g}^{2}+\left(\operatorname{tr}_{g} \Pi\right)^{2}=0
\end{array}
$$

where $g$ and $\Pi$ represent the induced Riemannian metric and the second fundamental form of $M$ respectively, whereas $S_{g}$ is the scalar curvature of $(M, g, \Pi)$. In the case where $\Pi \equiv 0$ the Cauchy data set is said to be time symmetric and the system above reduces to the vanishing of the scalar curvature. Therefore Theorem 2 automatically provides a generalized gluing construction for non Ricci flat initial data sets, in the spirit of [8].

\section{Geometric construction}

The geometric construction we use here is essentially the same we used in [16], but in order to fix the notation it is useful to transfer it, paying attention to the appropriate adjustments which are needed in our construction.

Let $\left(K, g_{K}\right)$ be a $k$-dimensional Riemannian manifold isometrically embedded in both the $n$-dimensional Riemannian manifolds $\left(M_{1}, g_{1}\right)$ and $\left(M_{2}, g_{2}\right)$, through the maps

$$
\iota_{i}: K \hookrightarrow M_{i} \quad i=1,2
$$

We assume that the isometric map $\iota_{1}^{-1} \circ \iota_{2}: \iota_{1}(K) \rightarrow \iota_{2}(K)$ extends to a diffeomorphism between the normal bundles of $\iota_{i}(K)$ in $\left(M_{i}, g_{i}\right), i=1,2$. We further assume that both the metrics $g_{1}$ and $g_{2}$ have zero constant scalar curvature. In this section our aim is to perform a generalized connected sum (or fiber sum) of the differentiable structures of $M_{1}$ and $M_{2}$ along the submanifold $K$. At the same time 
we construct on the new manifold $M=M_{1} \sharp_{K} M_{2}$ a family of metrics $\left(g_{\varepsilon}\right)_{\varepsilon \in(0,1)}$, whose scalar curvature is close to zero in a suitable sense.

For a fixed $\varepsilon \in(0,1)$, we describe the generalized connected sum construction and the definition of the metric $g_{\varepsilon}$ in a local coordinate system, the fact that this construction yields a globally defined metric will follow at once.

Let $U^{k}$ be an open set of $\mathbb{R}^{k}, B^{m-k}$ the $(m-k)$-dimensional open ball $(m-k \geq 3)$. For $i=1,2, F_{i}: U^{k} \times B^{m-k} \rightarrow W_{i} \subset M_{i}$ given by

$$
F_{i}(z, x):=\exp _{(z, 0)}^{M_{i}}(x)
$$

defines local Fermi coordinates near the coordinate patches $F_{i}(\cdot, 0)(U) \subset \iota_{i}(K) \subset$ $M_{i}$. In these coordinates, the metric $g_{i}$ can be decomposed as

$$
g_{i}(z, x)=g_{j l}^{(i)} d z^{j} \otimes d z^{l}+g_{\alpha \beta}^{(i)} d x^{\alpha} \otimes d x^{\beta}+g_{j \alpha}^{(i)} d z^{j} \otimes d x^{\alpha}
$$

and it is well known that, in this coordinate system,

$$
g_{\alpha \beta}^{(i)}=\delta_{\alpha \beta}+\mathcal{O}\left(|x|^{2}\right) \quad \text { and } \quad g_{j \alpha}^{(i)}=\mathcal{O}(|x|)
$$

In order to perform the identification between $W_{1}$ and $W_{2}$ and in order to glue the metrics together and define $g_{\varepsilon}$, we partially change the coordinate system, by setting

$$
\begin{array}{ll}
x=\varepsilon \mathrm{e}^{-t} \cdot \theta & \text { on } F_{1}^{-1}\left(W_{1}\right) \\
x=\varepsilon \mathrm{e}^{t} \cdot \theta & \text { on } F_{2}^{-1}\left(W_{2}\right)
\end{array}
$$

for $\varepsilon \in(0,1), \log \varepsilon<t<-\log \varepsilon, \theta \in S^{m-k-1}$.

Using these changes of coordinates the expressions of the two metrics $g_{1}$ and $g_{2}$ on $U^{k} \times A\left(\varepsilon^{2}, 1\right)$, where $A\left(\varepsilon^{2}, 1\right)$ is the annulus $\left\{\varepsilon^{2}<|x|<1\right\}$ become respectively

$$
\begin{aligned}
g_{1}(z, t, \theta)= & g_{i j}^{(1)} d z^{i} \otimes d z^{j} \\
& +u_{\varepsilon}^{(1)} \frac{4}{n-2}\left[\left(d t \otimes d t+g_{\lambda \mu}^{(1)} d \theta^{\lambda} \otimes d \theta^{\mu}\right)+g_{t \theta}^{(1)} d t \ltimes d \theta\right] \\
& +g_{i t}^{(1)} d z^{i} \otimes d t+g_{i \lambda}^{(1)} d z^{i} \otimes d \theta^{\lambda}
\end{aligned}
$$

and

$$
\begin{aligned}
g_{2}(z, t, \theta)= & g_{i j}^{(2)} d z^{i} \otimes d z^{j} \\
& +u_{\varepsilon}^{(2)} \frac{4}{n-2}\left[\left(d t \otimes d t+g_{\lambda \mu}^{(2)} d \theta^{\lambda} \otimes d \theta^{\mu}\right)+g_{t \theta}^{(2)} d t \ltimes d \theta\right] \\
& +g_{i t}^{(2)} d z^{i} \otimes d t+g_{i \lambda}^{(2)} d z^{i} \otimes d \theta^{\lambda}
\end{aligned}
$$

where by the compact notation $g_{t \theta} d t \ltimes d \theta$ we indicate the general component of the normal metric tensor (that is, it involves $d t \otimes d t, d \theta^{\lambda} \otimes d \theta^{\mu}$ and $d t \otimes d \theta^{\lambda}$ components). 
Observe that for $j=1,2$ we have

$$
\begin{array}{ll}
g_{\lambda \mu}^{(j)}=\mathcal{O}(1) & g_{t \theta}^{(j)}=\mathcal{O}\left(|x|^{2}\right) \\
g_{i t}^{(j)}=\mathcal{O}\left(|x|^{2}\right) & g_{i \lambda}^{(j)}=\mathcal{O}\left(|x|^{2}\right)
\end{array}
$$

and

$$
u_{\varepsilon}^{(1)}(t)=\varepsilon^{\frac{n-2}{2}} \mathrm{e}^{-\frac{n-2}{2} t} \quad \text { and } \quad u_{\varepsilon}^{(2)}(t)=\varepsilon^{\frac{n-2}{2}} \mathrm{e}^{\frac{n-2}{2} t}
$$

We choose a cut-off function $\zeta:(\log \varepsilon,-\log \varepsilon) \rightarrow[0,1]$ to be a nonincreasing smooth function which is identically equal to 1 in $(\log \varepsilon,-1]$ and 0 in $[1,-\log \varepsilon)$ and we choose another cut-off function $\eta:(\log \varepsilon,-\log \varepsilon) \rightarrow[0,1]$ to be a nonincreasing smooth function which is identically equal to 1 in $(\log \varepsilon,-\log \varepsilon-1]$ and which satisfies $\lim _{t \rightarrow-\log \varepsilon} \eta=0$. Using these two cut-off functions, we can define a new normal conformal factor $u_{\varepsilon}$ by

$$
u_{\varepsilon}(t):=\eta(t) u_{\varepsilon}^{(1)}(t)+\eta(-t) u_{\varepsilon}^{(2)}(t)
$$

and the metric $g_{\varepsilon}$ by

$$
\begin{aligned}
g_{\varepsilon}(z, t, \theta):= & \left(\zeta g_{i j}^{(1)}+(1-\zeta) g_{i j}^{(2)}\right) d z^{i} \otimes d z^{j} \\
& +u_{\varepsilon}^{\frac{4}{n-2}}\left[d t \otimes d t+\left(\zeta g_{\lambda \mu}^{(1)}+(1-\zeta) g_{\lambda \mu}^{(2)}\right) d \theta^{\lambda} \otimes d \theta^{\mu}\right. \\
& \left.+\left(\zeta g_{t \theta}^{(1)}+(1-\zeta) g_{t \theta}^{(2)}\right) d t \ltimes d \theta\right] \\
& +\left(\zeta g_{i t}^{(1)}+(1-\zeta) g_{i t}^{(2)}\right) d z^{i} \otimes d t \\
& +\left(\zeta g_{i \lambda}^{(1)}+(1-\zeta) g_{i \lambda}^{(2)}\right) d z^{i} \otimes d \theta^{\lambda}
\end{aligned}
$$

Closer inspection of this expression shows that the metric $g_{\varepsilon}$ (whose definition can be obviously completed by setting $g_{\varepsilon} \equiv g_{1}$ and $g_{\varepsilon} \equiv g_{2}$ out of the polyneck) is a Riemannian metric which is globally defined on the manifold $M$.

In the following we also need to consider some slight conformal (Sect. 5) and non conformal (Sect. 6) perturbations of the approximate solution metrics $g_{\varepsilon}$ 's away from the gluing locus. However, since such adjustments do not modify at all the linear analysis, we prefer to introduce them later, for sake of simplicity.

Following [16] it is easy to obtain the estimate for the scalar curvature of the approximate solution metric.

Proposition 3. (Estimate of the scalar curvature) There exists a constant $C>0$ independent of $\varepsilon \in(0,1)$ such that

$$
\left|S_{g_{\varepsilon}}\right| \leq C \cdot \varepsilon^{-1}(\operatorname{ch} t)^{1-n}
$$

for $|t| \leq|\log \varepsilon|$. 
Another useful tool we can recover from [16] is the expression for the $g_{\varepsilon}$-Laplacian on the polyneck

$$
\begin{aligned}
\Delta_{g_{\varepsilon}}= & u_{\varepsilon}^{-\frac{4}{n-2}}\left[\partial_{t}^{2}+(n-2) \operatorname{th}\left(\frac{n-2}{2} t\right) \partial_{t}+\Delta_{S^{n-1}}^{(\theta)}\right. \\
& \left.+u_{\varepsilon}^{\frac{4}{n-2}} \Delta_{K}^{(z)}+\mathcal{O}(|x|) \Phi\left(\nabla, \nabla^{2}\right)\right]
\end{aligned}
$$

where $\Phi\left(\nabla, \nabla^{2}\right)$ is a nonlinear differential operator involving first order and second order partial derivatives with respect to $t, \theta^{\lambda}$ and $z^{j}$ and whose coefficients are bounded uniformly on the polyneck, as $\varepsilon \in(0,1)$.

\section{Analysis of a linear operator}

Our aim is now to solve the Yamabe equation

$$
\Delta_{g_{\varepsilon}} u+c_{m} S_{g_{\varepsilon}} u=c_{m} S u^{\frac{m+2}{m-2}}
$$

where $c_{m}=-(m-2) / 4(m-1)$ and $S=S(\varepsilon)$ is a suitable constant. If $u$ is a solution to this equation, then the metric $\bar{g}_{\varepsilon}=u^{4 /(m-2)} g_{\varepsilon}$ has constant scalar curvature equal to $S$. Therefore, when we solve the Eq. (7), we also want to guarantee that $S$ tends to zero as $\varepsilon$ goes to zero, in order to prove the Theorem 1.

Since we want to preserve the structure of the two initial metrics far away from the gluing locus, we are looking for a conformal factor $u$ which is as close to 1 as we want. For this reason it is natural to set $u=1+v$ and to consider the equation

$$
\begin{aligned}
\Delta_{g_{\varepsilon}} v & =c_{m}\left(S-S_{g_{\varepsilon}}\right)+c_{m}\left(S-S_{g_{\varepsilon}}\right) v+\frac{4 c_{m}}{m-2} S v+c_{m} S f(v) \\
& =: F_{\varepsilon}(v)
\end{aligned}
$$

where $f(v)=(1+v)^{\frac{m+2}{m-2}}-1-\frac{m+2}{m-2} v$.

As already mentioned, the natural linearized operator to consider in a scalar flat context turns out to be the Lapalcian $\Delta_{g_{\varepsilon}}$. Since we want to invert it, we are forced to work orthogonally to the space of constant functions. Another problem is that on the generalized connected sum the first nonzero eigenvalue of $\Delta_{g_{\varepsilon}}$ tends to zero as $\varepsilon$ tends to zero. Roughly speaking, such an eigenvalue is produced by a function which takes approximately the value 1 on $M_{1}$ and -1 on $M_{2}$ (since $\operatorname{vol}_{g_{1}}\left(M_{1}\right)=1=\operatorname{vol}_{g_{2}}\left(M_{2}\right)$ ) and interpolates smoothly between these two values on the polyneck. As $\varepsilon$ tends to zero, the generalized connected sum degenerates into the two initial manifolds and the eigenfunction described above converges to a function which is the constant 1 on $M_{1}$ and the constant -1 on $M_{2}$. The corresponding eigenvalue is forced to tend to 0 in the limit. Notice that this reasoning can be made precise by adapting the argument presented in the appendix of [9]. Because of this fact it is not possible to provide (in natural function spaces) an a priori estimate which is uniformly bounded with respect to the parameter $\varepsilon$ for solutions of the linearized equation $\Delta_{g_{\varepsilon}} u=f$. Therefore we will adopt the following strategy: we first produce an approximate nonconstant eigenfunction $\beta_{\varepsilon}$ as 
explained above, then we obtain an $\varepsilon$-uniform a priori estimate for solutions to the projected linearized equation

$$
\Delta_{g_{\varepsilon}} u=f-\lambda \beta_{\varepsilon}
$$

where $f$ is a function such that $\int_{M} f \mathrm{dvol}_{g_{\varepsilon}}=0$. Here we are looking for a suitable constant $\lambda$ (which roughly speaking is the projection of $f$ along $\beta_{\varepsilon}$ ) and a solution $u$ which, up to a constant, can be chosen such that $\int_{M} u \operatorname{dvol}_{g_{\varepsilon}}=0$.

Combining the a priori estimate for Eq. (9) and the estimate of the scalar curvature $S_{g_{\varepsilon}}$ obtained in Proposition 3 we will then be able to solve the (nonlinear) fixed point problem

$$
\Delta_{g_{\varepsilon}} v=F_{\varepsilon}(v)-\lambda_{F_{\varepsilon}(v)} \beta_{\varepsilon}
$$

The final step is then to discuss the conditions which ensure the vanishing of the rough projection of the error term $\lambda_{F_{\varepsilon}(v)}$, providing a solution to Eq. (8).

As suggested by the title, this section is devoted to the solution of the linear problem (9). To begin with, let us fix the functional setting by recalling the following result from [16]:

Proposition 4. (Local a priori estimate) Given $\gamma \in(0, n-2)$, there exist real numbers $\alpha_{1}=\alpha_{1}(n, \gamma)>0, \alpha_{2}=\alpha_{2}(n, \gamma)>0$ and a constant $C_{n, \gamma} \geq 0$ such that for all $\varepsilon \in\left(0, \mathrm{e}^{-\max \left\{\alpha_{1}, \alpha_{2}\right\}}\right)$ and all $v, f \in \mathcal{C}^{0}\left(T^{\varepsilon}\left(\alpha_{1}, \alpha_{2}\right)\right)$ satisfying $\Delta_{g_{\varepsilon}} v=f$, the following estimate holds

$$
\sup _{T^{\varepsilon}\left(\alpha_{1}, \alpha_{2}\right)}\left|\psi_{\varepsilon}^{\gamma} v\right| \leq C_{n, \gamma}\left[\sup _{T^{\varepsilon}\left(\alpha_{1}, \alpha_{2}\right)}\left|\psi_{\varepsilon}^{\gamma+2} f\right|+\sup _{\partial T^{\varepsilon}\left(\alpha_{1}, \alpha_{2}\right)}\left|\psi_{\varepsilon}^{\gamma} v\right|\right]
$$

where $T^{\varepsilon}(\rho, \sigma):=\{\log \varepsilon+\rho \leq t \leq-\log \varepsilon-\sigma\}$, for $\rho, \sigma>0$ and $\psi_{\varepsilon}$ is the distance function defined as

$$
\psi_{\varepsilon}:= \begin{cases}\varepsilon \operatorname{ch} t & \text { in } T^{\varepsilon}(1,1) \\ 1 & \text { in } M \backslash T^{\varepsilon}(0,0)\end{cases}
$$

which interpolates smoothly between these two definitions.

(Observe that the statement is true for any couple of sufficiently large real numbers $\left.\left(\alpha_{1}, \alpha_{2}\right)\right)$.

Having at hand this result and working orthogonally to the kernel of $\Delta_{g_{\varepsilon}}$, it becomes natural to consider the weighted Banach spaces of continuous functions defined by:

$$
\mathcal{C}_{\delta}^{0}(M):=\left\{v \in \mathcal{C}^{0}(M):\|v\|_{\mathcal{C}_{\delta}^{0}(M)}<+\infty \text { and } \int_{M} v \operatorname{dvol}_{g_{\varepsilon}}=0\right\}
$$

where $\|v\|_{\mathcal{C}_{\delta}^{0}(M)}:=\sup _{M}\left|\psi_{\varepsilon}^{\delta} v\right|$, and $\delta \in \mathbb{R}$ is the weight. In our context we consider functions $f \in \mathcal{C}_{\gamma+2}^{0}(M)$ and we look for solutions $u \in \mathcal{C}_{\gamma}^{0}(M), \gamma \in(0, n-2)$. 
Let us describe now more precisely the function $\beta_{\varepsilon}$. For the reasons explained above it is useful to think of it as an approximation of the degenerate eigenfunction of $\Delta_{g_{\varepsilon}}$, whose associated eigenvalue tends to 0 as $\varepsilon \rightarrow 0$. We simply define $\beta_{\varepsilon}$ as

$$
\beta_{\varepsilon}:=\chi_{1}-\chi_{2}
$$

where $\chi_{1}$ and $\chi_{2}$ are smooth monotone functions which verify

$$
\begin{aligned}
& \chi_{1}=\left\{\begin{array}{lll}
1 & \text { on } & M_{1} \backslash T^{\varepsilon}\left(\alpha_{1}, 0\right) \\
0 & \text { on } & M_{2} \backslash T^{\varepsilon}\left(0,-2 \log \varepsilon-\alpha_{1}-1\right)
\end{array}\right. \\
& \chi_{2}:= \begin{cases}1 & \text { on } M_{2} \backslash T^{\varepsilon}\left(0, \alpha_{2}\right) \\
0 & \text { on } M_{1} \backslash T^{\varepsilon}\left(-2 \log \varepsilon-\alpha_{2}-1,0\right)\end{cases}
\end{aligned}
$$

Since by hypothesis $\operatorname{vol}\left(M_{1}\right)=1=\operatorname{vol}\left(M_{2}\right)$, it is always possible to choose two real numbers $\alpha_{1}$ and $\alpha_{2}$ such that

$$
\int_{M} \chi_{1}-\chi_{2} \operatorname{dvol}_{g_{\varepsilon}}=0
$$

This implies that the approximate eigenfunction $\beta_{\varepsilon}$ is orthogonal to the constants.

As a first step towards the solution of the problem (9) we will prove the following:

Lemma 1. Given a function $f \in \mathcal{C}_{\gamma+2}^{0}(M)$, it is possible to find a real number $\lambda=\lambda(f)$, an approximate solution $u \in \mathcal{C}_{\gamma}^{0}(M)$ and an error term $R \in \mathcal{C}_{\gamma+2}^{0}(M)$ that verify

$$
\Delta_{g_{\varepsilon}} u=f-\lambda \beta_{\varepsilon}+R
$$

Moreover $u, f$ and $R$ enjoy the following estimates:

$$
\begin{aligned}
\|u\|_{\mathcal{C}_{\gamma}^{0}(M)} & \leq A \cdot\|f\|_{\mathcal{C}_{\gamma+2}^{0}(M)} \\
|\lambda| & \leq B \cdot\|f\|_{\mathcal{C}_{\gamma+2}^{0}(M)} \\
\|R\|_{\mathcal{C}_{\gamma+2}^{0}(M)} & \leq C \cdot\|f\|_{\mathcal{C}_{\gamma+2}^{0}(M)} \cdot \varepsilon^{\beta \cdot \gamma}
\end{aligned}
$$

where the positive constants $A, B$ and $C$ depend on $K, n, \gamma, \alpha_{1}$ and $\alpha_{2}$, the weight $\gamma$ lies in $(0, n-2)$ and the real parameter $\beta$ can be chosen in $(0,1)$.

The proof of Lemma 1 consists in building an approximate solution $u$ to Eq. (9) and in estimating the remaining terms, collected in the error $R$. In order to do so, let us consider a non-negative smooth function $\chi_{P}$ such that the triple $\left\{\chi_{1}, \chi_{P}, \chi_{2}\right\}$ is a partition of the unity. It is useful to split $f$ into

$$
f=f \chi_{1}+f \chi_{P}+f \chi_{2}=f_{1}+f_{P}+f_{2}
$$

As a first step we want to build a good approximate solution on the polyneck. It is well known that the problem

$$
\begin{cases}\Delta_{g_{\varepsilon}} v=f_{P} & \text { on } T^{\varepsilon}\left(\alpha_{1}, \alpha_{2}\right) \\ v=0 & \text { on } \partial T^{\varepsilon}\left(\alpha_{1}, \alpha_{2}\right)\end{cases}
$$


admits a solution and we call it $\tilde{u}_{P}$. Moreover, if $f_{P}$ is continuous, so does $\tilde{u}_{P}$ and thanks to Proposition 4, if we choose large enough $\alpha_{1}$ and $\alpha_{2}$, we get immediately the estimate

$$
\left\|\tilde{u}_{P}\right\|_{\mathcal{C}_{\gamma}^{0}\left(T^{\varepsilon}\left(\alpha_{1}, \alpha_{2}\right)\right)} \leq A_{P} \cdot\left\|f_{P}\right\|_{\mathcal{C}_{\gamma+2}^{0}\left(T^{\varepsilon}\left(\alpha_{1}, \alpha_{2}\right)\right)}
$$

In fact the boundary conditions allow us to drop out the term $\left\|\tilde{u}_{P}\right\|_{\mathcal{C}_{\gamma}^{0}\left(\partial T^{\varepsilon}\left(\alpha_{1}, \alpha_{2}\right)\right)}$ in the estimate (11). Also notice that the positive constant $A_{P}$ only depends on $n, \gamma, \alpha_{1}$ and $\alpha_{2}$, so that the bound is uniform with respect to the parameter $\varepsilon$.

Let us smooth the polyneck solution just obtained, by defining $u_{P}:=\chi_{P} \tilde{u}_{P}$. As a consequence we have

$$
\begin{aligned}
\Delta_{g_{\varepsilon}} u_{P} & =\Delta_{g_{\varepsilon}} \tilde{u}_{P}-\Delta_{g_{\varepsilon}}\left(1-\chi_{P}\right) \tilde{u}_{P} \\
& =f_{P}-\Delta_{g_{\varepsilon}}\left(\chi_{1} \tilde{u}_{P}\right)-\Delta_{g_{\varepsilon}}\left(\chi_{2} \tilde{u}_{P}\right) \\
& =f_{P}-q_{1}-q_{2}
\end{aligned}
$$

where $q_{i}:=\Delta_{g_{\varepsilon}}\left(\chi_{i} \tilde{u}_{P}\right), i=1,2$.

As a second step we want now to construct approximate solutions on the pieces of $M$ coming from $M_{1}$ and $M_{2}$. To this purpose, let us consider, for $i=1,2$, the functions $\tilde{f}_{i}:=f_{i}+q_{i}$ and $\tilde{f}:=\tilde{f}_{1}+\tilde{f}_{2}$. Since $\int_{M} f \mathrm{dvol}_{g_{\varepsilon}}=0$, it is easy to check that $\int_{M} \tilde{f} \mathrm{dvol}_{g_{\varepsilon}}=0$. We also set $h_{i}:=\tilde{f}_{i}+(-1)^{i} \lambda \chi_{i}$ for $i=1,2$ and $h:=h_{1}+h_{2}=\tilde{f}-\lambda \beta_{\varepsilon}$. Obviously we have $\int_{M} h \operatorname{dvol}_{g_{\varepsilon}}=0$ and $\int_{M} h_{1} \operatorname{dvol}_{g_{\varepsilon}}=$ $-\int_{M} h_{2} \operatorname{dvol}_{g_{\varepsilon}}$.

Moreover

$$
\int_{M} h_{1} \operatorname{dvol}_{g_{\varepsilon}}-\int_{M} h_{2} \operatorname{dvol}_{g_{\varepsilon}}=\int_{M} \tilde{f}_{1}-\tilde{f}_{2} \operatorname{dvol}_{g_{\varepsilon}}-\lambda \int_{M} \chi_{1}+\chi_{2} \operatorname{dvol}_{g_{\varepsilon}}
$$

Hence, by setting

$$
\lambda:=\frac{\int_{M} \tilde{f}_{1}-\tilde{f}_{2} \mathrm{dvol}_{g_{\varepsilon}}}{\int_{M} \chi_{1}+\chi_{2} \operatorname{dvol}_{g_{\varepsilon}}}
$$

it follows at once that $\int_{M} h_{i} \operatorname{dvol}_{g_{\varepsilon}}=0, i=1,2$. Notice that the definition (18) allows us to think of $\lambda$ as the rough projection of $f$ along $\beta_{\varepsilon}$.

By slight modifications of very classical results (see [2]) we are allowed to consider solutions $\tilde{u}_{i}, i=1,2$ to the problems

$$
\Delta_{g_{i}} \tilde{u}_{i}=h_{i}-b_{i} \delta_{K}
$$

where $\delta_{K}$ is the Dirac distribution supported on the submanifold $K$ and the constants $b_{i}, i=1,2$ are forced to be

$$
b_{i}=\frac{\int_{M_{i}} h_{i} \mathrm{dvol}_{g_{i}}}{\operatorname{vol}_{g_{K}}(K)}
$$

It is rather simple to describe how for example the function $\tilde{u}_{1}$ approximately looks like, in fact we can write (notice that the following remarks still hold for $i=2$ ) 


$$
\Delta_{g_{1}} \tilde{u}_{1}=h_{1}+\frac{1}{V_{1}} \int_{M} h_{1} \operatorname{dvol}_{g_{1}}-\frac{1}{V_{1}} \int_{M} h_{1} \operatorname{dvol}_{g_{1}}-b_{1} \delta_{K}
$$

where $V_{1}$ is the short notation for $\operatorname{vol}_{g_{1}}\left(M_{1}\right)$. To proceed it is useful to consider the splitting $\tilde{u}_{1}=\bar{u}_{1}+\hat{u}_{1}$ where

$$
\left\{\begin{array}{l}
\Delta_{g_{1}} \bar{u}_{1}=h_{1}-\frac{1}{V_{1}} \int_{M} h_{1} \mathrm{dvol}_{g_{1}} \\
\Delta_{g_{1}} \hat{u}_{1}=\frac{1}{V_{1}} \int_{M} h_{1} \operatorname{dvol}_{g_{1}}-b_{1} \delta_{K}
\end{array}\right.
$$

We can think of $\bar{u}_{1}$ as the finite part and of $\hat{u}_{1}$ as the pure Green function part of $\tilde{u}_{1}$. In particular $\hat{u}_{1}$ has the following shape in a neighborhood of $K$ :

$$
\hat{u}_{1}=\Omega_{n, K} \cdot \int_{M} h_{1} \operatorname{dvol}_{g_{1}} \cdot\left[|x|^{2-n}+\mathcal{O}\left(|x|^{3-n}\right)\right]
$$

where $\Omega_{n, K}:=\left[\operatorname{vol}_{g_{K}}(K) \cdot(n-2) \cdot \omega_{n-1}\right]^{-1}$.

In order to estimate $\hat{u}_{1}$ it is useful to remember that, on the region $T^{\varepsilon}(0,0) \backslash$ $T^{\varepsilon}\left(\alpha_{1}, 0\right)$, the definition of the metric $g_{\varepsilon}$ implies that:

$$
\sqrt{g_{\varepsilon}}=\sqrt{g_{1}}+\mathcal{O}\left(\mathrm{e}^{(n-2) t}\right)
$$

Hence, thanks to the fact that $\int_{M} h_{1} \mathrm{dvol}_{g_{\varepsilon}}=0$, we can write

$$
\begin{aligned}
\int_{M_{1}} h_{1} \operatorname{dvol}_{g_{1}} & =\int_{M_{1}} h_{1} \operatorname{dvol}_{g_{1}}-\int_{M_{1}} h_{1} \operatorname{dvol}_{g_{\varepsilon}} \\
& =\int_{T^{\varepsilon}(0,0) \backslash T^{\varepsilon}\left(\alpha_{1}, 0\right)} h_{1}\left(\sqrt{g_{1}}-\sqrt{g_{\varepsilon}}\right) d z^{1} \ldots d z^{k} d t d \theta^{1} \ldots d \theta^{n-1}
\end{aligned}
$$

In the following, to keep short the notations, we will set

$$
\rho_{1, \varepsilon}:=\frac{\sqrt{g_{1}}-\sqrt{g_{\varepsilon}}}{\sqrt{g_{1}}}
$$

and we will indicate the volume element $\left(\sqrt{g_{1}}-\sqrt{g_{\varepsilon}}\right) d z^{1} \ldots d z^{k} d t d \theta^{1} \ldots d \theta^{n-1}$ by $\rho_{1, \varepsilon} \mathrm{dvol}_{g_{1}}$.

Let us recall that we have by definition $h_{1}=f_{1}+q_{1}-\lambda \chi_{1}$. Concerning the piece coming from $f_{1}$ it is straightforward to check that there exists a positive constant $\hat{A}_{K, n, \gamma, \alpha_{1}}^{\prime}$ such that

$$
\int_{T^{\varepsilon}(0,0) \backslash T^{\varepsilon}\left(\alpha_{1}+1,0\right)} f_{1} \rho_{1, \varepsilon} \operatorname{dvol}_{g_{1}} \leq \hat{A}_{K, n, \gamma, \alpha_{1}}^{\prime} \cdot\|f\|_{\mathcal{C}_{\gamma+2}^{0}(M)} \cdot \varepsilon^{n-2}
$$

To analyze the contribution of $q_{1}:=\Delta_{g_{\varepsilon}}\left(\chi_{1} \tilde{u}_{P}\right)$ it is convenient to write explicitly

$$
q_{1}=\left(\Delta_{g_{\varepsilon}} \chi_{1}\right) \tilde{u}_{P}+2 g_{\varepsilon}\left(d \chi_{1}, d \tilde{u}_{P}\right)+\chi_{1}\left(\Delta_{g_{\varepsilon}} \tilde{u}_{P}\right)
$$


Thanks to Proposition 4 and using the fact that $\Delta_{g_{\varepsilon}} \tilde{u}_{P}=f_{P}$, it is easy to see that there exists a positive constant $\hat{A}_{K, n, \gamma, \alpha_{1}}^{\prime \prime}$ such that

$$
\begin{aligned}
& \int_{T^{\varepsilon}\left(\alpha_{1}, 0\right) \backslash T^{\varepsilon}\left(\alpha_{1}+1,0\right)}\left(\Delta_{g_{\varepsilon}} \chi_{1}\right) \tilde{u}_{P}+\chi_{1}\left(\Delta_{g_{\varepsilon}} \tilde{u}_{P}\right) \quad \rho_{1, \varepsilon} \operatorname{dvol}_{g_{1}} \\
& \leq \hat{A}_{K, n, \gamma, \alpha_{1}}^{\prime \prime} \cdot\|f\|_{\mathcal{C}_{\gamma+2}^{0}(M)} \cdot \varepsilon^{n-2}
\end{aligned}
$$

To treat the remaining term it is convenient to integrate by parts, then using the fact that $\partial_{t} \chi_{1}$ vanish on $\partial\left[T^{\varepsilon}\left(\alpha_{1}, 0\right) \backslash T^{\varepsilon}\left(\alpha_{1}+1,0\right)\right]$ and Proposition 4 again, we deduce that

$$
\int_{T^{\varepsilon}\left(\alpha_{1}, 0\right) \backslash T^{\varepsilon}\left(\alpha_{1}+1,0\right)} 2 g_{\varepsilon}\left(d \chi_{1}, d \tilde{u}_{P}\right) \rho_{1, \varepsilon} \operatorname{dvol}_{g_{1}} \leq \hat{A}_{K, n, \gamma, \alpha_{1}}^{\prime \prime \prime} \cdot\|f\|_{\mathcal{C}_{\gamma+2}^{0}(M)} \cdot \varepsilon^{n-2}
$$

for some positive constant $\hat{A}_{K, n, \gamma, \alpha_{1}}^{\prime \prime \prime}$. Notice that a similar estimate also follows from the fact that, up to a careful choice of the cut off $\chi_{1}$, the term $g_{\varepsilon}\left(d \chi_{1}, d \tilde{u}_{P}\right)$ enjoys the following inequality

$$
\left|g_{\varepsilon}\left(d \chi_{1}, d \tilde{u}_{P}\right)\right| \leq C_{m, \gamma, \alpha_{1}} \cdot\left\|f_{P}\right\|_{\mathcal{C}_{\gamma+2}^{0}\left(T^{\varepsilon}\left(\alpha_{1}, \alpha_{2}\right)\right)}
$$

In fact, adapting to the Riemannian setting the very classical gradient estimate for bounded solutions of the Poisson equation [5] and recalling that $\tilde{u}_{P}$ is a bounded solution of $\Delta_{g_{\varepsilon}} \tilde{u}_{P}=f_{P}$ on the domain $D_{\alpha_{1}}:=T^{\varepsilon}\left(\alpha_{1}, 0\right) \backslash T^{\varepsilon}\left(\alpha_{1}+1,0\right)$, we get the bound

$$
\operatorname{dist}\left(y, \partial D_{\alpha_{1}}\right) \cdot\left|d \tilde{u}_{P}\right|(y) \leq C_{n, \alpha_{1}} \cdot\left[\left\|\tilde{u}_{P}\right\|_{\mathcal{C}^{0}\left(D_{\alpha_{1}}\right)}+\left\|f_{P}\right\|_{\mathcal{C}^{0}\left(D_{\alpha_{1}}\right)}\right]
$$

where $y$ is a point in $D_{\alpha_{1}}$ and $\operatorname{dist}\left(y, \partial D_{\alpha_{1}}\right)$ represents the distance from $y$ to the boundary of $D_{\alpha_{1}}$. Having this at hand we immediately get

$$
\left|g_{\varepsilon}\left(d \chi_{1}, d \tilde{u}_{P}\right)\right|(y) \leq C_{m, \gamma, \alpha_{1}} \cdot\left[\left\|\tilde{u}_{P}\right\|_{\mathcal{C}_{\gamma}^{0}\left(D_{\alpha_{1}}\right)}+\left\|f_{P}\right\|_{\mathcal{C}_{\gamma+2}^{0}\left(D_{\alpha_{1}}\right)}\right] \cdot \frac{\left|d \chi_{1}\right|(y)}{\operatorname{dist}\left(y, \partial D_{\alpha_{1}}\right)}
$$

If $\chi_{1}$ is sufficiently smooth, then the last factor in the right hand side is bounded in $D_{\alpha_{1}}$ and Proposition 4 yields the estimate (22).

The definition of the rough projection $\lambda$ (18) obviously implies (modulo the same computation on $M_{2}$ ) the estimate (15) in the statement of Lemma 1 and, as a consequence, an analog of the estimates above for the term $-\lambda \chi_{1}$ which appears in the expression of $\int_{M_{1}} h_{1} \mathrm{dvol}_{g_{1}}$.

Hence, recalling the expression (21) of $\hat{u}_{1}$, we conclude that there exists a positive constant $\hat{A}_{K, n, \gamma, \alpha_{1}}^{1}$ such that

$$
\left|\hat{u}_{1}\right| \leq \hat{A}_{K, n, \gamma, \alpha_{1}}^{1} \cdot\|f\|_{\mathcal{C}_{\gamma+2}^{0}(M)} \cdot \mathrm{e}^{(n-2) t}
$$

Also notice that formula (21) implies at once analogous estimates for the derivatives of $\hat{u}_{1}$ with respect to the variables $t, \theta^{\lambda}, z^{i}$, for $\lambda=1, \ldots, n-1$ and $i=1, \ldots, k$. 
Let us look now to the finite part of $\tilde{u}_{1}$, namely $\bar{u}_{1}$. If we define $\bar{h}_{1}$ as

$$
\bar{h}_{1}:=h_{1}-\frac{1}{V_{1}} \int_{M_{1}} h_{1} \operatorname{dvol}_{g_{1}}
$$

then $\Delta_{g_{1}} \bar{u}_{1}=\bar{h}_{1}$ and the classical Green representation formula (see for example [2]) for $\bar{u}_{1}$ automatically yields the estimate

$$
\left\|\bar{u}_{1}\right\|_{\mathcal{C}^{1}\left(M_{1}\right)} \leq\left\|\bar{h}_{1}\right\|_{\mathcal{C}^{0}\left(M_{1}\right)}
$$

Applying the remark (22) and Proposition 4, we deduce that there exists a positive constant $\bar{A}_{K, n, \gamma, \alpha_{1}}^{\prime}$ such that

$$
\left|q_{1}\right| \leq \bar{A}_{K, n, \gamma, \alpha_{1}}^{\prime} \cdot\|f\|_{\mathcal{C}_{\gamma+2}^{0}(M)}
$$

Hence, also the $\mathcal{C}^{0}$ norm of $\bar{h}_{1}$ is bounded by

$$
\left\|\bar{h}_{1}\right\|_{\mathcal{C}^{0}\left(M_{1}\right)} \leq \bar{A}_{K, n, \gamma, \alpha_{1}}^{\prime \prime} \cdot\|f\|_{\mathcal{C}_{\gamma+2}^{0}(M)}
$$

for some positive constant $\bar{A}_{K, n, \gamma, \alpha_{1}}^{\prime \prime}$. This implies that there exists $\bar{A}_{K, n, \gamma, \alpha_{1}}^{1}>0$ such that

$$
\left|\bar{u}_{1}\right| \leq \bar{A}_{K, n, \gamma, \alpha_{1}}^{1} \cdot\|f\|_{\mathcal{C}_{\gamma+2}^{0}(M)}
$$

and the same is true for the derivatives of $\bar{u}_{1}$ with respect to the variables $t, \theta^{\lambda}, z^{i}$, for $\lambda=1, \ldots, n-1$ and $i=1, \ldots, k$.

To summarize, we obtain from (25) and (23) that there exists a positive constant $A_{K, n, \gamma, \alpha_{1}}^{1}$ such that the function $\tilde{u}_{1}=\bar{u}_{1}+\hat{u}_{1}$ is bounded by

$$
\left|\tilde{u}_{1}\right| \leq A_{K, n, \gamma, \alpha_{1}}^{1} \cdot\|f\|_{\mathcal{C}_{\gamma+2}^{0}(M)}
$$

and the same is true for its derivatives with respect to the variables $t, \theta^{\lambda}, z^{i}$, for $\lambda=1, \ldots, n-1$ and $i=1, \ldots, k$.

Following the same strategy it is straightforward to obtain a similar result for a function $\tilde{u}_{2}$, which is the analog of $\tilde{u}_{1}$ on $M_{2}$. Now, using $\tilde{u}_{1}, \tilde{u}_{2}$ and $u_{P}$ (which is nothing but the polyneck solution $\tilde{u}_{P}$ smoothed down), we are ready to produce the approximate solution $u$ of Lemma 1 . To do that, we introduce, for $\beta \in(0,1)$, the smooth monotone cut off functions $\phi_{1}$ and $\phi_{2}$ which verify

$$
\begin{aligned}
& \phi_{1}=\left\{\begin{array}{lll}
1 & \text { on } & M_{1} \backslash T^{\varepsilon}(-\beta \log \varepsilon, 0) \\
0 & \text { on } & M_{2} \backslash T^{\varepsilon}(0,-(2-\beta) \log \varepsilon-1)
\end{array}\right. \\
& \phi_{2}= \begin{cases}1 & \text { on } M_{2} \backslash T^{\varepsilon}(0,-\beta \log \varepsilon) \\
0 & \text { on } M_{1} \backslash T^{\varepsilon}(-(2-\beta) \log \varepsilon-1,0)\end{cases}
\end{aligned}
$$

To complete the description of the cut-offs we assume that $\phi_{1}$ interpolates smoothly between $(1-\beta) \log \varepsilon$ and $(1-\beta) \log \varepsilon+1$ and $\phi_{2}$ does the same between 
$-(1-\beta) \log \varepsilon-1$ and $-(1-\beta) \log \varepsilon$. Notice that for small enough $\varepsilon$, we have that supp $\left(d \phi_{i}\right) \subset T^{\varepsilon}\left(\alpha_{1}, \alpha_{2}\right)$, for $i=1,2$ (in the following we will always assume that).

Let us define now the approximate solution as

$$
u:=\phi_{1} \tilde{u}_{1}+u_{P}+\phi_{2} \tilde{u}_{2}
$$

Notice that the estimate (17) and the estimate (26), with its analog for $\tilde{u}_{2}$, imply at once the estimate (14) in the statement of Lemma 1, namely there exists a positive constant $A_{K, n, \gamma, \alpha_{1}, \alpha_{2}}$ such that

$$
\|u\|_{\mathcal{C}_{\gamma}^{0}(M)} \leq A_{K, n, \gamma, \alpha_{1}, \alpha_{2}} \cdot\|f\|_{\mathcal{C}_{\gamma+2}^{0}(M)}
$$

To define the error term $R$ of Lemma 1, we compute

$$
\begin{aligned}
\Delta_{g_{\varepsilon}} u= & \Delta_{g_{\varepsilon}} u_{P}+\Delta_{g_{\varepsilon}}\left(\phi_{1} \tilde{u}_{1}\right)+\Delta_{g_{\varepsilon}}\left(\phi_{2} \tilde{u}_{2}\right) \\
= & f_{P}-q_{1}-q_{2} \\
& +\phi_{1}\left(h_{1}-b_{1} \delta_{K}\right)+\left(\Delta_{g_{\varepsilon}} \phi_{1}\right) \tilde{u}_{1}+g_{\varepsilon}\left(d \phi_{1}, d \tilde{u}_{1}\right) \\
& +\phi_{2}\left(h_{2}-b_{2} \delta_{K}\right)+\left(\Delta_{g_{\varepsilon}} \phi_{2}\right) \tilde{u}_{2}+g_{\varepsilon}\left(d \phi_{2}, d \tilde{u}_{2}\right) \\
= & f-\lambda \beta_{\varepsilon} \\
& +\left(\Delta_{g_{\varepsilon}} \phi_{1}\right) \tilde{u}_{1}+g_{\varepsilon}\left(d \phi_{1}, d \tilde{u}_{1}\right) \\
& +\left(\Delta_{g_{\varepsilon}} \phi_{2}\right) \tilde{u}_{2}+g_{\varepsilon}\left(d \phi_{2}, d \tilde{u}_{2}\right)
\end{aligned}
$$

At this point it is quite natural to define $E_{i}:=\left(\Delta_{g_{\varepsilon}} \phi_{i}\right) \tilde{u}_{i}+g_{\varepsilon}\left(\nabla \phi_{i}, \nabla \tilde{u}_{i}\right), i=1,2$ and $R:=E_{1}+E_{2}$, so that $u, \lambda$ and $R$ satisfy the Eq. (13)

$$
\Delta_{g_{\varepsilon}} u=f-\lambda \beta_{\varepsilon}+R
$$

The last task in order to complete the proof of the Lemma 1 is to provide $R$ with the estimate (16). Without loss of generality, let us look for example at the error term $E_{1}$.

First notice that since supp $\left(\Delta_{g_{\varepsilon}} \phi_{1}\right)$ and supp $\left(d \phi_{1}\right)$ are both included in $[(1-\beta)$ $\log \varepsilon,(1-\beta) \log \varepsilon+1]$, the term $E_{1}$ is supported here as well. Considering this fact and the estimates obtained for $\tilde{u}_{1}$ and its derivatives, it is straightforward to deduce that, for a suitable positive constant $C_{K, n, \gamma, \alpha_{1}}^{1}$,

$$
\left\|E_{1}\right\|_{\mathcal{C}_{\gamma+2}^{0}(M)} \leq C_{K, n, \gamma, \alpha_{1}}^{1} \cdot\|f\|_{\mathcal{C}_{\gamma+2}^{0}(M)} \cdot \varepsilon^{\beta \gamma}
$$

This estimate and its counterpart for $E_{2}$ imply the estimate (16) for $R$ and this completes the proof of Lemma 1.

Now we want to look at the Eq. (9). The idea is to solve it by means of an iteration process. We start by setting $f^{(0)}:=f$ and thanks to Lemma 1 we obtain a triple $\left(\lambda^{(0)}, u^{(0)}, R^{(0)}\right)$ satisfying

$$
\Delta_{g_{\varepsilon}} u^{(0)}=f^{(0)}-\lambda^{(0)} \beta_{\varepsilon}+R^{(0)}
$$

and the estimates (14), (15) and (16). Then, setting $f^{(1)}:=-R^{(0)}$, we find another triple $\left(\lambda^{(1)}, u^{(1)}, R^{(1)}\right)$ with the same properties as the first one and so on. In general, 
for every $j \in \mathbb{N}$, we have $f^{(j)}:=-R^{(j-1)}$ and a triple $\left(\lambda^{(j)}, u^{(j)}, R^{(j)}\right)$ satisfying the equation

$$
\Delta_{g_{\varepsilon}} u^{(j)}=f^{(j)}-\lambda^{(j)} \beta_{\varepsilon}+R^{(j)}
$$

and the estimates (14), (15) and (16) of Lemma 1.

Taking the sum of the Eq. (31) we have that, for every $N \in \mathbb{N}$

$$
\begin{aligned}
\Delta_{g_{\varepsilon}} \sum_{j=0}^{N} u^{(j)} & =\sum_{j=0}^{N} f^{(j)}-\beta_{\varepsilon} \sum_{j=0}^{N} \lambda^{(j)}+\sum_{j=0}^{N} R^{(j)} \\
& =f-\beta_{\varepsilon} \sum_{j=0}^{N} \lambda^{(j)}+R^{(N)}
\end{aligned}
$$

We can rephrase this by saying

$$
\Delta_{g_{\varepsilon}} v^{(N)}=f-\mu^{(N)} \beta_{\varepsilon}+R^{(N)}
$$

where

$$
v^{(N)}:=\sum_{j=0}^{N} u^{(j)} \text { and } \mu^{(N)}:=\sum_{j=0}^{N} \lambda^{(j)}
$$

From the estimates of Lemma 1 it easily follows that

$$
\begin{aligned}
\left\|f^{(j)}\right\|_{\mathcal{C}_{\gamma+2}^{0}(M)}=\left\|R^{(j-1)}\right\|_{\mathcal{C}_{\gamma+2}^{0}(M)} & \leq C \cdot\left\|f^{(j-1)}\right\|_{\mathcal{C}_{\gamma+2}^{0}(M)} \cdot \varepsilon^{\beta \gamma} \\
& \leq\left(C \varepsilon^{\beta \gamma}\right)^{j} \cdot\|f\|_{\mathcal{C}_{\gamma+2}^{0}(M)} \\
\left\|u^{(j)}\right\|_{\mathcal{C}_{\gamma}^{0}(M)} & \leq A \cdot\left\|f^{(j)}\right\|_{\mathcal{C}_{\gamma+2}^{0}(M)} \\
& \leq A \cdot\left(C \varepsilon^{\beta \gamma}\right)^{j} \cdot\|f\|_{\mathcal{C}_{\gamma+2}^{0}(M)} \\
\left|\lambda^{(j)}\right| & \leq B \cdot\left\|f^{(j)}\right\|_{\mathcal{C}_{\gamma+2}^{0}(M)} \\
& \leq B \cdot\left(C \varepsilon^{\beta \gamma}\right)^{j} \cdot\|f\|_{\mathcal{C}_{\gamma+2}^{0}(M)}
\end{aligned}
$$

It is clear that, for sufficiently small $\varepsilon>0$, there exist a real number $\lambda \in \mathbb{R}$ and function $u \in \mathcal{C}_{\gamma}^{0}$ such that

$$
R^{(N)} \stackrel{\mathcal{C}_{\gamma+2}^{0}}{\longrightarrow} 0, \quad v^{(N)} \stackrel{\mathcal{C}_{\gamma}^{0}}{\longrightarrow} u, \quad \mu^{(N)} \longrightarrow \lambda
$$

Moreover there exist positive constants $A^{\prime}$ and $B^{\prime}$ depending on $K, n, \gamma, \alpha_{1}$ and $\alpha_{2}$ such that

$$
\|u\|_{\mathcal{C}_{\gamma}^{0}(M)} \leq A^{\prime}\|f\|_{\mathcal{C}_{\gamma+2}^{0}(M)} \text { and } \quad|\lambda| \leq B^{\prime}\|f\|_{\mathcal{C}_{\gamma+2}^{0}(M)}
$$


Hence

$$
\Delta_{g_{\varepsilon}} v^{(N)} \stackrel{\mathcal{C}_{\gamma+2}^{0}}{\longrightarrow} f-\lambda \beta_{\varepsilon} \quad \text { and } \quad v^{(N)} \stackrel{\mathcal{C}_{\gamma}^{0}}{\longrightarrow} u
$$

On the other hand we have that, for every $N \in \mathbb{N}$ and for every $\phi \in \mathcal{C}^{\infty}(M)$

$$
\int_{M} v^{(N)} \Delta_{g_{\varepsilon}} \phi \operatorname{dvol}_{g_{\varepsilon}}=\int_{M}\left(f-\mu^{(N)} \beta_{\varepsilon}+R^{(N)}\right) \phi \operatorname{dvol}_{g_{\varepsilon}}
$$

Hence, by taking the limit for $N \rightarrow+\infty$ we find, for every $\phi \in \mathcal{C}^{\infty}(M)$, the expression

$$
\int_{M} u \Delta_{g_{\varepsilon}} \phi \operatorname{dvol}_{g_{\varepsilon}}=\int_{M}\left(f-\lambda \beta_{\varepsilon}\right) \phi \operatorname{dvol}_{g_{\varepsilon}}
$$

In other words the following identity

$$
\Delta_{g_{\varepsilon}} u=f-\lambda \beta_{\varepsilon}
$$

holds in the sense of the distributions.

Thanks to the elliptic regularity, if we suppose $f \in \mathcal{C}^{0, \alpha}(M)$, then $u \in \mathcal{C}^{2, \alpha}$ and the expression above is a point-wise identity.

To conclude this section we summarize our results in the following

Proposition 5. Given a function $f \in \mathcal{C}_{\gamma+2}^{0}(M)$, it is possible to find a real number $\lambda$ and a function $u \in \mathcal{C}_{\gamma}^{0}(M)$ such that the equation

$$
\Delta_{g_{\varepsilon}} u=f-\lambda \beta_{\varepsilon}
$$

is satisfied in the sense of the distributions and the following estimates hold

$$
\begin{aligned}
\|u\|_{\mathcal{C}_{\gamma}^{0}(M)} & \leq A^{\prime} \cdot\|f\|_{\mathcal{C}_{\gamma+2}^{0}(M)} \\
|\lambda| & \leq B^{\prime} \cdot\|f\|_{\mathcal{C}_{\gamma+2}^{0}(M)}
\end{aligned}
$$

for suitable positive constants $A^{\prime}$ and $B^{\prime}$ depending on $K, n, \gamma, \alpha_{1}$ and $\alpha_{2}$.

Moreover, if $f \in \mathcal{C}^{0, \alpha}(M)$, then $u \in \mathcal{C}^{2, \alpha}(M)$ and the identity above holds point-wise.

\section{Nonlinear analysis: a fixed point argument}

The aim of this section is to solve the fixed point problem (10), namely

$$
\Delta_{g_{\varepsilon}} v=F_{\varepsilon}(v)-\lambda_{F_{\varepsilon}(v)} \beta_{\varepsilon}
$$

We will be able to do this using a fixed point theorem for contracting mappings, provided the $\mathcal{C}_{\gamma}^{0}(M)$-norm of $v$ is small enough. 
Before starting, let us remark that in the expression of $F_{\varepsilon}(v)(8)$ it is always possible to choose $S=S(\varepsilon, v)$ in such a way that $\int_{M} F_{\varepsilon} \mathrm{dvol}_{g_{\varepsilon}}=0$, in fact it is sufficient to set

$$
S:=\frac{\int_{M} S_{g_{\varepsilon}}(1+v) \mathrm{dvol}_{g_{\varepsilon}}}{\int_{M} 1+\frac{m+2}{m-2} v+f(v) \mathrm{dvol}_{g_{\varepsilon}}}
$$

Using the estimate of the scalar curvature (6) of Proposition 3 it is not hard to check that $S=\mathcal{O}\left(\varepsilon^{n-2}\right)$.

Observe that in Sects. 5 and 6, we will need to introduce some slight modifications of the approximate solution metrics $g_{\varepsilon}$ away from the gluing locus, in order to kill the rough projection $\lambda_{F_{\varepsilon}}(v)$. This will induce some small changes in the expression of $S_{g_{\varepsilon}}$ and consequently, at a first time, we will find an estimate for $S$ which is strictly worse than the one we claimed in the statement of Theorem 1. More precisely, it will turn out that $S$ is roughly estimated as $S=\mathcal{O}\left(\varepsilon^{(n-2) / 2}\right)$ instead of $S=\mathcal{O}\left(\varepsilon^{n-2}\right)$. However, the fixed point argument which we are going to describe now, will still hold in the modified context of Sects. 5 and 6, modulo a small adjustment which is explained below. Using then this fixed point result, we will obtain a solution $v_{\varepsilon}$ to the Eq. (10) in the new setting. Next, using the perturbations introduced in the approximate solution metrics and the fact that the Eq. (10) is verified in this new context, it will be possible to kill the rough projection $\lambda_{F_{\varepsilon}}\left(v_{\varepsilon}\right)$ improving at the same time the estimate for $S$, getting $S=\mathcal{O}\left(\varepsilon^{n-2}\right)$, as claimed. Having this in mind, we prefer, for sake of simplicity, to prove the existence of a fixed point for the problem above in the current situation.

To begin with, let us introduce the maps

$$
\begin{aligned}
F_{\varepsilon}: \mathcal{C}_{\gamma}^{0}(M) & \longrightarrow \mathcal{C}_{\gamma+2}^{0}(M) \\
v & \longmapsto F_{\varepsilon}(v) \\
G_{\varepsilon}: \mathcal{C}_{\gamma+2}^{0}(M) & \longrightarrow \mathcal{C}_{\gamma}^{0}(M) \\
w & \longmapsto G_{\varepsilon}(w)
\end{aligned}
$$

where $G_{\varepsilon}(w)$ is the unique solution to the equation $\Delta_{g_{\varepsilon}} G_{\varepsilon}(w)=w-\lambda_{w} \beta_{\varepsilon}$, with $\lambda_{w}$ given as in Proposition 5. Notice that $\lambda_{w}$ is also unique in reason of the estimates contained in Proposition 5.

At this point it is quite natural to define the map

$$
\begin{aligned}
P_{\varepsilon}: \mathcal{C}_{\gamma}^{0}(M) & \longrightarrow \mathcal{C}_{\gamma}^{0}(M) \\
v & \longmapsto G_{\varepsilon} \circ F_{\varepsilon}(v)
\end{aligned}
$$

Now we can state the following:

Lemma 2. For $\gamma \in(0,1 / 2)$ and for sufficiently small $\varepsilon>0$ there exists a radius $r_{\varepsilon}:=\varepsilon^{2 \gamma}$ such that $P_{\varepsilon}\left(B_{\gamma}\left(r_{\varepsilon}\right)\right) \subset B_{\gamma}\left(r_{\varepsilon}\right)$, where $B_{\gamma}\left(r_{\varepsilon}\right):=\left\{u \in \mathcal{C}_{\gamma}^{0}(M)\right.$ : $\left.\|u\|_{\mathcal{C}_{\gamma}^{0}(M)} \leq r_{\varepsilon}\right\}$. In other words:

$$
\|v\|_{\mathcal{C}_{\gamma}^{0}(M)} \leq r_{\varepsilon} \Longrightarrow\left\|P_{\varepsilon}(v)\right\|_{\mathcal{C}_{\gamma}^{0}(M)} \leq r_{\varepsilon}
$$


In order to prove the statement, we start by observing that the choice of $S$ and the estimate of $S_{g_{\varepsilon}}$ obtained in Proposition 3 imply at once that $F_{\varepsilon}(v) \in \mathcal{C}_{\gamma+2}^{0}$, for $v \in B_{\gamma}\left(r_{\varepsilon}\right)$. Then, using the estimate (33) of Proposition 5 we immediately get

$$
\left\|P_{\varepsilon}(v)\right\|_{\mathcal{C}_{\gamma}^{0}(M)} \leq D \cdot\left\|F_{\varepsilon}(v)\right\|_{\mathcal{C}_{\gamma+2}^{0}(M)}
$$

for a suitable positive constant $D$.

Now, we have to estimate the right hand side of the above expression. Recalling the definition of $r_{\varepsilon}$ and the estimate (6), we compute

$$
\begin{aligned}
\left|F_{\varepsilon}(v) \psi_{\varepsilon}^{\gamma+2}\right| \leq & c_{1}|S| \psi_{\varepsilon}^{\gamma+2}+c_{2}\left|S_{g_{\varepsilon}}\right| \psi_{\varepsilon}^{\gamma+2}+c_{3}|S| \psi_{\varepsilon}^{\gamma+2}|v| \\
& +c_{4}\left|S_{g_{\varepsilon}}\right| \psi_{\varepsilon}^{\gamma+2}|v|+c_{5}|S||f(v)| \psi_{\varepsilon}^{\gamma+2} \\
\leq & c_{6} \varepsilon^{n-2}+c_{7}\left[\varepsilon^{n-2}+\varepsilon^{1+\gamma}\right]+c_{8} \varepsilon^{n-2+\gamma} \\
& +c_{9}\left[\varepsilon^{n-2}+\varepsilon^{1+\gamma}\right] \varepsilon^{\gamma}+c_{10} \varepsilon^{n-2+2 \gamma} \\
\leq & c_{11} \varepsilon^{n-2}+c_{12} \varepsilon^{1+\gamma} \\
\leq & {\left[c_{13} \varepsilon^{n-2-2 \gamma}+\varepsilon^{1-\gamma}\right] r_{\varepsilon} }
\end{aligned}
$$

for suitable positive constants $c_{j}$ 's. Therefore, the Lemma is proved, provided $\gamma \in(0,1 / 2)$.

Let us insist on the fact that in the following sections we will consider slight modifications of the approximate solutions. As anticipated, this will forces us to modify the choice of $S$. This new $S$ can only be estimated, at a first time as a $\mathcal{O}\left(\varepsilon^{(n-2) / 2}\right)$. Nevertheless, up to choose $\gamma$ in a smaller range $(\gamma \in(0,1 / 4))$, the statement of the Lemma 2 and its consequences remain true.

At this point, our aim is to prove that the sequence defined as

$$
v^{j}:=P_{\varepsilon}^{j}(0)
$$

for every $j \in \mathbb{N}$, converges to a function $v_{\varepsilon} \in B_{\gamma}\left(r_{\varepsilon}\right)$ with respect to the norm $\|\cdot\|_{\mathcal{C}_{\gamma}^{0}(M)}$.

Since we want to use a contraction mapping argument, we need to provide an estimate for $\left\|P_{\varepsilon}(u)-P_{\varepsilon}(v)\right\|_{\mathcal{C}_{\gamma}^{0}(M)}$ in terms of $\|u-v\|_{\mathcal{C}_{\gamma}^{0}(M)}$, where $u, v \in B_{\gamma}\left(r_{\varepsilon}\right)$. In fact, since $0 \in B_{\gamma}\left(r_{\varepsilon}\right)$, all the terms belong to $B_{\gamma}\left(r_{\varepsilon}\right)$, because of Lemma 2 .

First notice that

$$
\Delta_{g_{\varepsilon}}\left(P_{\varepsilon}(u)-P_{\varepsilon}(v)\right)=F_{\varepsilon}(u)-F_{\varepsilon}(v)-\left(\lambda_{F_{\varepsilon}(u)}-\lambda_{F_{\varepsilon}(v)}\right) \beta_{\varepsilon}
$$

Next we observe that $f \mapsto \lambda_{f}$, where $\lambda_{f}$ is the rough projection defined in Proposition 5, is a linear map, hence

$$
\lambda_{F_{\varepsilon}(u)}-\lambda F_{\varepsilon}(v)=\lambda F_{\varepsilon}(u)-F_{\varepsilon}(v)
$$

As a consequence of the estimate (33) we obtain

$$
\left\|P_{\varepsilon}(u)-P_{\varepsilon}(v)\right\|_{\mathcal{C}_{\gamma}^{0}(M)} \leq C_{0} \cdot\left\|F_{\varepsilon}(u)-F_{\varepsilon}(v)\right\|_{\mathcal{C}_{\gamma+2}^{0}(M)}
$$

for some suitable positive constant $C_{0}$. 
Since the function $f$ which appears in the definition of $F_{\varepsilon}(v)$ satisfies the following inequality

$$
|f(u)-f(v)| \leq\left[C_{1}(|u|+|v|)+C_{2}\left(|u|^{\frac{4}{m-2}}-|v|^{\frac{4}{m-2}}\right)\right]|u-v|
$$

for suitable positive constants $C_{1}$ and $C_{2}$, we can proceed with the estimate of the term $F_{\varepsilon}(u)-F_{\varepsilon}(v)$. The condition $\gamma \in(0,1 / 2)$ is a sufficient condition to ensure that

$$
\begin{aligned}
\psi_{\varepsilon}^{\gamma+2}\left|F_{\varepsilon}(u)-F_{\varepsilon}(v)\right| \leq & \psi_{\varepsilon}^{\gamma+2}\left[c_{m}|S||u-v|+c_{m}\left|S_{g_{\varepsilon}}\right||u-v|+\frac{|S|}{m-1}|u-v|\right] \\
& +\psi_{\varepsilon}^{\gamma+2} c_{m}|S|\left[C_{1}(|u|+|v|)\right. \\
& \left.+C_{2}\left(|u|^{\frac{4}{m-2}}-|v|^{\frac{4}{m-2}}\right)\right]|u-v| \\
\leq & C_{3} \varepsilon \cdot\|u-v\|_{\mathcal{C}_{\gamma}^{0}(M)} \\
& +C_{4} \varepsilon^{n-2} \cdot\left[\|u\|_{\mathcal{C}_{\gamma}^{0}(M)}+\|v\|_{\mathcal{C}_{\gamma}^{0}(M)}\right] \cdot\|u-v\|_{\mathcal{C}_{\gamma}^{0}(M)} \\
& +C_{5} \varepsilon^{n-2} \cdot\left[\|u\|_{\mathcal{C}_{\gamma}^{0}(M)}^{\frac{4}{m-2}}+\|v\|_{\mathcal{C}_{\gamma}^{0}(M)}^{\frac{4}{m-2}}\right] \cdot\|u-v\|_{\mathcal{C}_{\gamma}^{0}(M)}
\end{aligned}
$$

for suitable positive $C_{j}$ 's. (Again, the condition on $\gamma$ becomes slightly different for the metrics we will consider in the next sections, namely $\gamma \in(0,1 / 4))$.

Hence, for $u, v \in B_{\gamma}\left(r_{\varepsilon}\right)$ and $\varepsilon>0$ small enough, we obtain the inequality

$$
\left\|P_{\varepsilon}(u)-P_{\varepsilon}(v)\right\|_{\mathcal{C}_{\gamma}^{0}(M)} \leq C_{6} \varepsilon \cdot\|u-v\|_{\mathcal{C}_{\gamma}^{0}(M)}
$$

Therefore, if we choose two integers $p \leq q$, we have that

$$
\begin{aligned}
\left\|v^{q}-v^{p}\right\|_{\mathcal{C}_{\gamma}^{0}(M)} & \leq \sum_{j=1}^{p-q}\left\|v^{p+j}-v^{p+j-1}\right\|_{\mathcal{C}_{\gamma}^{0}(M)} \\
& \leq\left(C_{6} \varepsilon\right)^{p} \cdot \sum_{j=0}^{+\infty}\left(C_{6} \varepsilon\right)^{j} \cdot\left\|v^{1}-v^{0}\right\|_{\mathcal{C}_{\gamma}^{0}(M)}
\end{aligned}
$$

Therefore, the sequence $\left(v^{j}\right)$ is a Cauchy sequence and it must converge to a function $v_{\varepsilon} \in B_{\gamma}\left(r_{\varepsilon}\right)$ which is the fixed point of $P_{\varepsilon}$ in $B_{\gamma}\left(r_{\varepsilon}\right)$, namely

$$
P_{\varepsilon}\left(v_{\varepsilon}\right)=v_{\varepsilon}
$$

Recalling the definition of $P_{\varepsilon}$, it is straightforward to see that $v_{\varepsilon}$ is a solution to the problem (10)

$$
\Delta_{g_{\varepsilon}} v_{\varepsilon}=F_{\varepsilon}\left(v_{\varepsilon}\right)-\lambda_{F_{\varepsilon}\left(v_{\varepsilon}\right)} \beta_{\varepsilon}
$$

Notice that by means of a classical boot strap argument one can prove that $v_{\varepsilon}$ is actually a smooth function.

To conclude this section, let us remark that since $v_{\varepsilon}$ has been found by means of a contraction mapping argument, it also depends continuously on the data of our problem. 


\section{Vanishing of the rough projection $\lambda_{F_{\varepsilon}\left(v_{\varepsilon}\right)}$}

In this section we want to discuss the vanishing of $\lambda_{F_{\varepsilon}}\left(v_{\varepsilon}\right)$. This is the last step needed to complete the proof of Theorem 1 . In fact we recall that our main purpose is to produce a solution to the Eq. (8). We also recall that $F_{\varepsilon}(v)$ represents the error term in this equation, and that we can think of $\lambda_{F_{\varepsilon}(v)}$ as the rough projection of the error term along the first nonconstant eigenfunction $\beta_{\varepsilon}$ of the linearized operator $\Delta_{g_{\varepsilon}}$. At the moment we have produced a solution $v_{\varepsilon}$ to the Eq. (10), if we are able to ensure the vanishing of $\lambda_{F_{\varepsilon}\left(v_{\varepsilon}\right)}$, then we have finished. To do so, we consider some slight conformal modifications of the initial metrics $g_{1}$ and $g_{2}$ supported away from the gluing locus.

More precisely let $\bar{w}_{1}$ and $\bar{w}_{2}$ be two smooth functions supported on $M_{1} \backslash W_{1}$ and $M_{2} \backslash W_{2}$ respectively (with the notation introduced in Sect. 2). Then, we set

$$
\begin{aligned}
& w_{1}:=a \varepsilon^{(n-2) / 2} \cdot \bar{w}_{1} \\
& w_{2}:=b \varepsilon^{(n-2) / 2} \cdot \bar{w}_{2}
\end{aligned}
$$

where $a$ and $b$ are real parameters. Having defined $w_{1}$ and $w_{2}$, we introduce the modified metrics

$$
\begin{aligned}
& \tilde{g}_{1}:=\left(1+w_{1}\right)^{\frac{4}{m-2}} g_{1} \\
& \tilde{g}_{2}:=\left(1+w_{2}\right)^{\frac{4}{m-2}} g_{2}
\end{aligned}
$$

Using $\tilde{g}_{1}$ and $\tilde{g}_{2}$ instead of $g_{1}$ and $g_{2}$ in the geometric construction presented in Sect. 2 we obtain a family of modified approximate solution metrics $\left(\tilde{g}_{\varepsilon}\right)_{\varepsilon \in(0,1)}$ and it is easy to check that the linear analysis remains unchanged for these new metrics. Namely Proposition 5 still holds with $\tilde{g}_{\varepsilon}$ instead of $g_{\varepsilon}$ (because of the support of $\left.w_{i}, i=1,2\right)$. Also observe that, because of the smallness of the modification introduced, it is always possible to chose $\alpha_{1}$ and $\alpha_{2}$ in the definition of $\chi_{1}$ and $\chi_{2}$ such that

$$
\int_{M} \chi_{1}-\chi_{2} \operatorname{dvol}_{\tilde{g}_{\varepsilon}}=0
$$

It turns out that the scalar curvature of $\tilde{g}_{i}$ is supported on supp $\left(\bar{w}_{i}\right)$, and that it is given by

$$
S_{\tilde{g}_{i}}=\left(1+w_{i}\right)^{-\frac{m+2}{m-2}} \cdot \Delta_{g_{i}} w_{i}
$$

for $i=1,2$, then $S_{\tilde{g}_{i}}=\mathcal{O}\left(\varepsilon^{(n-2) / 2}\right)$. From this it is easy to deduce an analog of Proposition 3 for the scalar curvature $S_{\tilde{g}_{\varepsilon}}$. More precisely $S_{\tilde{g}_{\varepsilon}}$ enjoys an estimate similar to the one of $S_{g_{\varepsilon}}$ (namely the estimate (6)) on the polyneck, whereas it turns out to be a $\mathcal{O}\left(\varepsilon^{(n-2) / 2}\right)$ on the supports of the $w_{i}$ 's. As a consequence, we also deduce a rough estimate for $S$, namely $S=\mathcal{O}\left(\varepsilon^{(n-2) / 2}\right)$. In the following, once a new fixed point is found in the modified setting, we will also improve this estimate, in order to get $S=\mathcal{O}\left(\varepsilon^{n-2}\right)$, as required in the statement of Theorem 1 . 
As already mentioned, up to choose $\gamma \in(0,1 / 4)$, we can reproduce with slight modifications the fixed point argument of Sect. 4 , in order to obtain a solution to the equation

$$
\Delta_{\tilde{g}_{\varepsilon}} v_{\varepsilon}=\tilde{F}_{\varepsilon}\left(v_{\varepsilon}\right)-\lambda_{\tilde{F}_{\varepsilon}\left(v_{\varepsilon}\right)} \beta_{\varepsilon}
$$

where the explicit expression for $\tilde{F}_{\varepsilon}\left(v_{\varepsilon}\right)$ is

$$
\tilde{F}_{\varepsilon}\left(v_{\varepsilon}\right)=c_{m} S\left[1+\frac{m+2}{m-2} v_{\varepsilon}+f\left(v_{\varepsilon}\right)\right]-c_{m} S_{\tilde{g}_{\varepsilon}}\left[1+v_{\varepsilon}\right]
$$

and $S$ is now given by

$$
\begin{aligned}
S & =\frac{\int_{M} S_{\tilde{g}_{\varepsilon}}\left(1+v_{\varepsilon}\right) \operatorname{dvol}_{\tilde{g}_{\varepsilon}}}{\int_{M} 1+\frac{m+2}{m-2} v_{\varepsilon}+f\left(v_{\varepsilon}\right) \operatorname{dvol}_{\tilde{g}_{\varepsilon}}} \\
& =\frac{\int_{M}\left(S_{\tilde{g}_{1}}+S_{\tilde{g}_{\varepsilon}}+S_{\tilde{g}_{2}}\right)\left(1+v_{\varepsilon}\right) \operatorname{dvol}_{\tilde{g}_{\varepsilon}}}{\operatorname{vol}_{\tilde{g}_{\varepsilon}}(M)}+\mathcal{O}\left(\varepsilon^{(n-2) / 2+\gamma}\right)
\end{aligned}
$$

Also notice that the fact that $v_{\varepsilon}$ lies in $B_{\gamma}\left(r_{\varepsilon}\right)$ implies at once the estimate (1) which appears in the statement of Theorem 1 .

The task is now to show that $\lambda_{\tilde{F}_{\varepsilon}\left(v_{\varepsilon}\right)}$ can be chosen to be zero. Since all the quantities which appear in the expression (47) depend smoothly on the real parameters $a$ and $b$ introduced in the definitions of the $w_{i}$ 's, our goal is achieved if we prove that we can control the sign of the rough projection $\lambda_{\tilde{F}_{\varepsilon}\left(v_{\varepsilon}\right)}$ by means of $a$ and $b$.

Following the proof of Proposition 5, we can think of $\lambda_{\tilde{F}_{\varepsilon}\left(v_{\varepsilon}\right)}$ as a sum of the series

$$
\lambda_{\tilde{F}_{\varepsilon}\left(v_{\varepsilon}\right)}=\sum_{j=0}^{\infty} \lambda_{\tilde{F}_{\varepsilon}\left(v_{\varepsilon}\right)}^{(j)}
$$

where the real numbers $\lambda_{\tilde{F}_{\varepsilon}\left(v_{\varepsilon}\right)}^{(j)}$ are built on as in the mentioned proposition and consequently enjoy the estimate

$$
\left|\lambda{\overline{\tilde{F}_{\varepsilon}\left(v_{\varepsilon}\right)}}_{(j)}\right| \leq B \cdot\left(C \varepsilon^{\beta \gamma}\right)^{j} \cdot\left\|\tilde{F}_{\varepsilon}\left(v_{\varepsilon}\right)\right\|_{\mathcal{C}_{\gamma+2}^{0}(M)}
$$

where $B$ and $C$ are positive constants depending on $K, n, \gamma, \alpha_{1}$ and $\alpha_{2}$ and the real parameter $\beta$ lies in $(0,1)$. As indicated by this estimate, the leading term in the expression for $\lambda_{\tilde{F}_{\varepsilon}\left(v_{\varepsilon}\right)}$ is given by the first summand. More precisely, using the explicit definition of the $\lambda_{\tilde{F}_{\varepsilon}\left(v_{\varepsilon}\right)}^{(j)}$, s, it is not hard to show that, for a suitable choice of the parameter $\beta$, all these terms are $o\left(\varepsilon^{n-2}\right)$, for $j \geq 1$. We deduce that the sign of the rough projection is determined by the one of $\lambda_{\tilde{F}_{\varepsilon}\left(v_{\varepsilon}\right)}^{(0)}$, which is explicitly given by

$$
\begin{aligned}
\lambda_{\tilde{F}_{\varepsilon}\left(v_{\varepsilon}\right)}^{(0)}= & \frac{1}{\int_{M} \chi_{1}+\chi_{2} \operatorname{dvol}_{\tilde{g}_{\varepsilon}}} \int_{M} \tilde{F}_{\varepsilon}\left(v_{\varepsilon}\right)\left(\chi_{1}-\chi_{2}\right) \operatorname{dvol}_{\tilde{g}_{\varepsilon}} \\
& +\frac{1}{\int_{M} \chi_{1}+\chi_{2} \operatorname{dvol}_{\tilde{g}_{\varepsilon}}} \int_{M} \Delta_{g_{\varepsilon}}\left(\chi_{1} \tilde{u}_{P}^{\varepsilon}\right)-\Delta_{g_{\varepsilon}}\left(\chi_{2} \tilde{u}_{P}^{\varepsilon}\right) \operatorname{dvol}_{\tilde{g}_{\varepsilon}}
\end{aligned}
$$


where $\tilde{u}_{P}^{\varepsilon}$ is the solution of the problem

$$
\begin{cases}\Delta_{g_{\varepsilon}} \tilde{u}_{P}^{\varepsilon}=\chi_{P} \cdot \tilde{F}_{\varepsilon}\left(v_{\varepsilon}\right) & \text { on } T^{\varepsilon}\left(\alpha_{1}, \alpha_{2}\right) \\ \tilde{u}_{P}^{\varepsilon}=0 & \text { on } \partial T^{\varepsilon}\left(\alpha_{1}, \alpha_{2}\right)\end{cases}
$$

Let us focus now on the term $\int_{M} \tilde{F}_{\varepsilon}\left(v_{\varepsilon}\right)\left(\chi_{1}-\chi_{2}\right)$ dvol $_{g_{\varepsilon}}$ which appears in (53). Recalling the expression for $\tilde{F}_{\varepsilon}\left(v_{\varepsilon}\right)$, it becomes

$$
\begin{gathered}
c_{m} S \int_{M} \frac{m+2}{m-2} v_{\varepsilon}\left(\chi_{1}-\chi_{2}\right)+f\left(v_{\varepsilon}\right)\left(\chi_{1}-\chi_{2}\right) \operatorname{dvol}_{\tilde{g}_{\varepsilon}} \\
-c_{m} \int_{M}\left(S_{\tilde{g}_{1}}+S_{g_{\varepsilon}}+S_{\tilde{g}_{2}}\right)\left(1+v_{\varepsilon}\right)\left(\chi_{1}-\chi_{2}\right) \operatorname{dvol}_{\tilde{g}_{\varepsilon}}
\end{gathered}
$$

It is not hard to show that

$$
\begin{aligned}
& \int_{M} S_{g_{\varepsilon}}\left(1+v_{\varepsilon}\right) \chi_{1} \operatorname{dvol}_{\tilde{g}_{\varepsilon}}=-4 n \operatorname{vol}_{g_{K}}(K) \omega_{n-1} \cdot \varepsilon^{n-2}+\mathcal{O}\left(\mathrm{e}^{-\alpha_{1}} \varepsilon^{n-2}\right) \\
& \int_{M} S_{g_{\varepsilon}}\left(1+v_{\varepsilon}\right) \chi_{2} \operatorname{dvol}_{\tilde{g}_{\varepsilon}}=-4 n \operatorname{vol}_{g_{K}}(K) \omega_{n-1} \cdot \varepsilon^{n-2}+\mathcal{O}\left(\mathrm{e}^{-\alpha_{2}} \varepsilon^{n-2}\right)
\end{aligned}
$$

Moreover it is also straightforward to see that

$$
\begin{aligned}
& \int_{M} S_{\tilde{g}_{1}} \chi_{1} \operatorname{dvol}_{\tilde{g}_{\varepsilon}}=-\int_{M_{1}}\left|d w_{1}\right|_{g_{1}}^{2} \operatorname{dvol}_{g_{1}}+\mathcal{O}\left(\varepsilon^{\frac{3(n-2)}{2}}\right) \\
& \int_{M} S_{\tilde{g}_{2}} \chi_{2} \operatorname{dvol}_{\tilde{g}_{\varepsilon}}=-\int_{M_{2}}\left|d w_{2}\right|_{g_{2}}^{2} \operatorname{dvol}_{g_{2}}+\mathcal{O}\left(\varepsilon^{\frac{3(n-2)}{2}}\right)
\end{aligned}
$$

The estimate of the term $\int_{M}\left(S_{\tilde{g}_{1}} \chi_{1}+S_{\tilde{g}_{2}} \chi_{2}\right) v_{\varepsilon} \mathrm{dvol}_{\tilde{g}_{\varepsilon}}$ is more delicate. Let us look for example at the term $\int_{M} S_{\tilde{g}_{1}} v_{\varepsilon} \operatorname{dvol}_{\tilde{g}_{\varepsilon}}$. A direct computation shows that

$$
\int_{M} S_{\tilde{g}_{1}} v_{\varepsilon} \operatorname{dvol}_{\tilde{g}_{\varepsilon}}=\int_{M_{1}} w_{1}\left(\Delta_{g_{1}} v_{\varepsilon}\right) \operatorname{dvol}_{g_{1}}+\mathcal{O}\left(\varepsilon^{n-2+\gamma}\right)
$$

Using simple Taylor expansions we obtain (on the support of $w_{1}$ )

$$
\begin{aligned}
\Delta_{g_{1}} v_{\varepsilon}= & {\left[1+\frac{4}{m-2} w_{1}+\mathcal{O}\left(\varepsilon^{n-2}\right)\right] \cdot \Delta_{\tilde{g}_{\varepsilon}} v_{\varepsilon} } \\
& -2 g_{1}\left(d w_{1}, d v_{\varepsilon}\right)+2 w_{1} g_{1}\left(d w_{1}, d v_{\varepsilon}\right)+\mathcal{O}\left(\varepsilon^{\frac{3(n-2)}{2}}\right)
\end{aligned}
$$

Integrating against $w_{1}$ gives

$$
\int_{M} S_{\tilde{g}_{1}} v_{\varepsilon} \operatorname{dvol}_{\tilde{g}_{\varepsilon}}=\int_{M_{1}} w_{1} \tilde{F}_{\varepsilon}\left(v_{\varepsilon}\right) \operatorname{dvol}_{g_{1}}-\lambda_{\tilde{F}_{\varepsilon}\left(v_{\varepsilon}\right)} \cdot \int_{M_{1}} w_{1} \operatorname{dvol}_{g_{1}}+\mathcal{O}\left(\varepsilon^{n-2+\gamma}\right)
$$


Performing a rough estimate one can easily see that $\tilde{F}_{\varepsilon}\left(v_{\varepsilon}\right)=\mathcal{O}\left(\varepsilon^{(n-2) / 2}\right)$ on the support of $w_{1}$ and also $\lambda_{\tilde{F}_{\varepsilon}\left(v_{\varepsilon}\right)}=\mathcal{O}\left(\varepsilon^{(n-2) / 2}\right)$. Combining these facts with the computation above, we improve the rough estimate for $S$ and we immediately obtain $S=\mathcal{O}\left(\varepsilon^{n-2}\right)$.

Again, using the explicit formula for $\tilde{F}_{\varepsilon}$, one can refine (54) obtaining

$$
\int_{M} S_{\tilde{g}_{1}} v_{\varepsilon} \operatorname{dvol}_{\tilde{g}_{\varepsilon}}=c_{m} \int_{M_{1}}\left|d w_{1}\right|_{g_{1}}^{2} \operatorname{dvol}_{g_{1}}+\lambda_{\tilde{F}_{\varepsilon}\left(v_{\varepsilon}\right)} \cdot \mathcal{O}\left(\varepsilon^{\frac{n-2}{2}}\right)+\mathcal{O}\left(\varepsilon^{\frac{3(n-2)}{2}}\right)
$$

Collecting all the information obtained we deduce that

$$
\begin{aligned}
\int_{M} \tilde{F}_{\varepsilon}\left(v_{\varepsilon}\right) \cdot\left(\chi_{1}-\chi_{2}\right) \operatorname{dvol}_{\tilde{g}_{\varepsilon}}= & \left(c_{m}^{2}-c_{m}\right) \cdot \int_{M}\left|d w_{2}\right|_{g_{2}}^{2} \mathrm{dvol}_{g_{2}} \\
& -\left(c_{m}^{2}-c_{m}\right) \cdot \int_{M}\left|d w_{1}\right|_{g_{1}}^{2} \mathrm{dvol}_{g_{1}} \\
& +\lambda_{\tilde{F}_{\varepsilon}\left(v_{\varepsilon}\right)} \cdot \mathcal{O}\left(\varepsilon^{(n-2) / 2)}\right) \\
& +\mathcal{O}\left(\mathrm{e}^{-\alpha_{1}} \varepsilon^{n-2}\right)+\mathcal{O}\left(\mathrm{e}^{-\alpha_{2}} \varepsilon^{n-2}\right)
\end{aligned}
$$

If we write $\Delta_{g_{\varepsilon}}\left(\chi_{i} \tilde{u}_{P}^{\varepsilon}\right)=\chi_{i}\left(\Delta_{g_{\varepsilon}} \tilde{u}_{P}^{\varepsilon}\right)+2\left[g_{\varepsilon}\left(d \chi_{i}, d \tilde{u}_{P}^{\varepsilon}\right)+\tilde{u}_{P}^{\varepsilon}\left(\Delta_{g_{\varepsilon}} \chi_{i}\right)\right]-\tilde{u}_{P}^{\varepsilon}$ $\left(\Delta_{g_{\varepsilon}} \chi_{i}\right)$, then Green's formula implies, for $i=1,2$

$$
\int_{M} \Delta_{g_{\varepsilon}}\left(\chi_{i} \tilde{u}_{P}^{\varepsilon}\right) \operatorname{dvol}_{\tilde{g}_{\varepsilon}}=\int_{M} \chi_{1} \chi_{P} \tilde{F}_{\varepsilon}\left(v_{\varepsilon}\right) \operatorname{dvol}_{g_{\varepsilon}}-\int_{M} \tilde{u}_{P}^{\varepsilon}\left(\Delta_{g_{\varepsilon}} \chi_{i}\right) \operatorname{dvol}_{g_{\varepsilon}}
$$

Following the proof of Proposition 4 contained in [16] and taking into account the shape of $\tilde{F}_{\varepsilon}\left(v_{\varepsilon}\right)$, one gets the following bound for $\tilde{u}_{P}^{\varepsilon}$

$$
\left|\tilde{u}_{P}^{\varepsilon}\right| \leq C \cdot \varepsilon^{n-2} \cdot \psi_{\varepsilon}^{\gamma-(n-2)}
$$

where the positive constant $C$, does not depend on $\varepsilon$.

Using this fact it is straightforward to deduce that

$$
\begin{aligned}
& \int_{M} \Delta_{g_{\varepsilon}}\left(\chi_{1} \tilde{u}_{P}^{\varepsilon}\right) \operatorname{dvol}_{\tilde{g}_{\varepsilon}}=\mathcal{O}\left(\mathrm{e}^{-\alpha_{1}} \varepsilon^{n-2}\right) \\
& \int_{M} \Delta_{g_{\varepsilon}}\left(\chi_{i} \tilde{u}_{P}^{\varepsilon}\right) \operatorname{dvol}_{\tilde{g}_{\varepsilon}}=\mathcal{O}\left(\mathrm{e}^{-\alpha_{2}} \varepsilon^{n-2}\right)
\end{aligned}
$$

These estimates and the expression (55) imply that the sign of $\lambda_{\tilde{F}_{\varepsilon}\left(v_{\varepsilon}\right)}$ is determined, for small $\varepsilon$ and sufficiently large $\alpha_{1}$ and $\alpha_{2}$, by the term

$$
\left(c_{m}^{2}-c_{m}\right) \cdot \int_{M}\left|d w_{2}\right|_{g_{2}}^{2} \operatorname{dvol}_{g_{2}}-\left(c_{m}^{2}-c_{m}\right) \cdot \int_{M}\left|d w_{1}\right|_{g_{1}}^{2} \operatorname{dvol}_{g_{1}}
$$


Hence it is clear that if we move the real parameters $a$ and $b$ in the definition of the $w_{i}$ 's, the sign of the rough projection changes. Since the solution depends continuously on these parameters, we conclude that for a suitable choice of $a$ and $b$ the rough projection $\lambda_{\tilde{F}_{\varepsilon}\left(v_{\varepsilon}\right)}$ of the error term $\tilde{F}_{\varepsilon}\left(v_{\varepsilon}\right)$ along $\beta_{\varepsilon}$ is zero and Theorem 1 is proved.

\section{Getting $S=0$ : the non Ricci flat case}

In this section we will prove Theorem 2. As claimed in the statement, when both the initial metrics are scalar flat but non Ricci flat it is possible to construct a zero scalar curvature metrics on the generalized connected sum. The idea consists in doing slight non conformal modifications of the approximate solution metrics $g_{\varepsilon}$ 's away from the gluing locus. By means of these modifications it is possible to obtain at once the orthogonality to the constant functions of the new error term $\tilde{F}_{\varepsilon}\left(v_{\varepsilon}\right)$ and the vanishing of its rough projection along the approximate degenerate eigenfunction $\beta_{\varepsilon}$ of the linearized operator $\Delta_{\tilde{g}_{\varepsilon}}$. Remember that in the proof of Theorem 1, we used the nonzero constant scalar curvature $S=S\left(\varepsilon, v_{\varepsilon}\right)$ to insure the first condition and slight conformal modifications of the $g_{\varepsilon}$ 's to get the second one.

Let us describe the construction. Instead of the metrics $g_{\varepsilon}$ 's let us consider the new approximate solution metric

$$
\tilde{g}_{\varepsilon}(r, s)=g_{\varepsilon}+r h_{1}+s h_{2}
$$

where $h_{1}$ and $h_{2}$ are positive definite symmetric tensors supported respectively on the manifolds $M_{1}$ and $M_{2}$ away from the polyneck, and $r$ and $s$ are real parameters. Also remark that the $h_{i}$ 's are non conformal to $g_{i}$ 's, $i=1,2$.

The equation we need to solve as a first step is the following

$$
\Delta_{\tilde{g}_{\varepsilon}} v=\tilde{F}_{\varepsilon}(v, r, s)-\lambda_{\tilde{F}_{\varepsilon}(v, r, s)} \cdot \beta_{\varepsilon}
$$

where the new error term is given by $\tilde{F}_{\varepsilon}(v, r, s):=-c_{m} S_{\tilde{g}_{\varepsilon}}(1+v)$. Notice that this definition automatically imposes that the scalar curvature we are going to achieve is constant and equal to zero. Again we assume that $\int_{M} \beta_{\varepsilon} \operatorname{dvol}_{\tilde{g}_{\varepsilon}}=0$.

Once a solution $v_{\varepsilon}(r, s)$ is obtained, we will discuss the vanishing of the rough projection $\lambda_{\tilde{F}_{\varepsilon}\left(v_{\varepsilon}, r, s\right)}$. This will complete the proof of Theorem 2.

As in the previous case, we will be able to find a solution to the nonlinear problem (58) using a fixed point argument for contraction mapping which will produce a fixed point for the Eq. (58) as a solution of an iteration scheme.

Concerning the linear analysis, notice that the construction above allows us to use the results of Proposition 5, once the orthogonality of the error term $\tilde{F}_{\varepsilon}(v, r, s)$ to the constant functions is provided.

Let us therefore focus on the condition

$$
\int_{M} \tilde{F}_{\varepsilon}(v, r, s) \operatorname{dvol}_{\tilde{g}_{\varepsilon}}=0
$$

Since $\int_{M} S_{g_{\varepsilon}} \operatorname{dvol}_{\tilde{g}_{\varepsilon}}=\mathcal{O}\left(\varepsilon^{n-2}\right)$, we are allowed to choose the correction parameters of the same size, namely $r, s=\mathcal{O}\left(\varepsilon^{n-2}\right)$. 
Before starting the calculation, let us make some remarks about the scalar curvature of the metric $\tilde{g}_{\varepsilon}$, in order to get more information on $\tilde{F}_{\varepsilon}$. Since the supports of $S_{g_{\varepsilon}}, h_{1}$ and $h_{2}$ are disjoint and $S_{g_{1}}=0=S_{g_{2}}$, we can write, following [3]

$$
\begin{aligned}
S_{\tilde{g}_{\varepsilon}} & =S_{g_{\varepsilon}}+S_{g_{1}+r h_{1}}+S_{g_{2}+s h_{2}} \\
& =S_{g_{\varepsilon}}+r K_{1}+\mathcal{O}\left(r^{2}\right)+s K_{2}+\mathcal{O}\left(s^{2}\right)
\end{aligned}
$$

where

$$
K_{i}=\Delta_{g_{i}}\left(\operatorname{tr}_{g_{i}} h_{i}\right)+\delta_{g_{i}}\left(\delta_{g_{i}} h_{i}\right)+g_{i}\left(\operatorname{Ric}_{g_{i}}, h_{i}\right) \quad i=1,2
$$

In the formula above $\delta_{g_{i}}$ denotes the divergence of a symmetric tensor with respect to the metric $g_{i}$, and $\mathrm{Ric}_{g_{i}}$ is the Ricci tensor of the metric $g_{i}$, for $i=1,2$.

Integrating the $K_{i}$ 's over $M$, we obtain

$$
\begin{aligned}
\int_{M} K_{i} \operatorname{dvol}_{\tilde{g}_{\varepsilon}} & =\int_{M_{i}} K_{i} \operatorname{dvol}_{\tilde{g}_{i}} \\
& =\int_{M_{i}} g_{i}\left(\operatorname{Ric}_{g_{i}}, h_{i}\right) \operatorname{dvol}_{g_{i}}+\mathcal{O}\left(\varepsilon^{n-2}\right)
\end{aligned}
$$

where $\tilde{g}_{1}=g_{1}+r h_{1}$ and $\tilde{g}_{2}=g_{2}+s h_{2}$. Notice that, in the Ricci flat case, the integral above is zero and there is no chance to correct the term $\int_{M} S_{g_{\varepsilon}}(1+v) \operatorname{dvol}_{g_{\varepsilon}}$ in order to get the right orthogonality condition.

For $v \in B_{\gamma}\left(r_{\varepsilon}\right)$ (where $r_{\varepsilon}=\varepsilon^{2 \gamma}$, as in Sect. 4), let us define the map $G_{\varepsilon, v}(r, s)$ as follows:

$$
\begin{aligned}
G_{\varepsilon, v}(r, s):= & \int_{M} S_{\tilde{g}_{\varepsilon}}(1+v) \operatorname{dvol}_{\tilde{g}_{\varepsilon}} \\
= & \int_{M} S_{g_{\varepsilon}} \mathrm{dvol}_{g_{\varepsilon}}+r \int_{M_{1}} K_{1} \mathrm{dvol}_{g_{1}}+s \int_{M_{2}} K_{2} \mathrm{dvol}_{g_{2}} \\
& +L_{v}(r, s)+Q_{v}(r, s)
\end{aligned}
$$

where

$$
\begin{aligned}
L_{v}(r, s):= & \int_{M} S_{g_{\varepsilon}} v \operatorname{dvol}_{g_{\varepsilon}}+r \int_{M_{1}} K_{1} v \operatorname{dvol}_{g_{1}}+s \int_{M_{2}} K_{2} v \operatorname{dvol}_{g_{2}} \\
Q_{v}(r, s):= & \int_{M_{1}} S_{g_{1}+r h_{1}}(1+v) \operatorname{dvol}_{g_{1}}-r \int_{M_{1}} K_{1}(1+v) \operatorname{dvol}_{g_{1}} \\
& +\int_{M_{2}} S_{g_{2}+h_{2}}(1+v) \operatorname{dvol}_{g_{2}}-s \int_{M_{2}} K_{2}(1+v) \operatorname{dvol}_{g_{2}}+\mathcal{O}\left(\varepsilon^{2(n-2)}\right)
\end{aligned}
$$

Our next task is to describe the set where $G_{\varepsilon, v}(r, s)$ is zero. 
To proceed, let us consider the map

$$
H_{\varepsilon}(r, s):=G_{\varepsilon, v}(r, s)-L_{v}(r, s)-Q_{v}(r, s)
$$

In order to simplify the following computation, we can assume that the symmetric tensors $h_{1}$ and $h_{2}$ are chosen so that $\int_{M_{1}} K_{1} \mathrm{dvol}_{g_{1}}=1=\int_{M_{2}} K_{2} \mathrm{dvol}_{g_{2}}$. We can further assume, without loss of generality, that $\int_{M} S_{g_{\varepsilon}} \mathrm{dvol}_{g_{\varepsilon}}<0$ and since $\int_{M} S_{g_{\varepsilon}} \mathrm{dvol}_{g_{\varepsilon}}=\mathcal{O}\left(\varepsilon^{n-2}\right)$ we can set, up to normalize, $\int_{M} S_{g_{\varepsilon}} \mathrm{dvol}_{g_{\varepsilon}}=-\varepsilon^{n-2}$. With these assumption, the expression for $H_{\varepsilon}$ becomes then the following

$$
H_{\varepsilon}(r, s)=-\varepsilon^{n-2}+r+s
$$

The set where $H_{\varepsilon}$ vanishes is given by $\left\{(r, s) \in \mathbb{R}^{2} \mid r+s=\varepsilon^{n-2}\right\}$. We will show that the set where $G_{\varepsilon, v}$ vanishes is uniformly close to the set $\left\{H_{\varepsilon}=0\right\}$, for $v \in B_{\gamma}\left(r_{\varepsilon}\right)$.

Since $r, s=\mathcal{O}\left(\varepsilon^{n-2}\right)$ it is easy to see that there exist two positive constants $C_{L}$ and $C_{Q}$ such that, for all $v \in B_{\gamma}\left(r_{\varepsilon}\right)$

$$
\begin{aligned}
& L_{v}(r, s) \leq C_{L} \cdot \varepsilon^{n-2+\gamma} \\
& Q_{v}(r, s) \leq C_{Q} \cdot \varepsilon^{2(n-2)+\gamma}
\end{aligned}
$$

In particular, for an arbitrarily small fixed constant $c>0$ and sufficiently small $\varepsilon>0$ we have

$$
\begin{aligned}
& \left|L_{v}(r, s)\right| \leq(c / 2) \varepsilon^{n-2} \\
& \left|Q_{v}(r, s)\right| \leq(c / 2) \varepsilon^{n-2}
\end{aligned}
$$

At this point, it is immediate to check that for all $v \in B_{\gamma}\left(r_{\varepsilon}\right)$

$$
\begin{aligned}
\left\{G_{\varepsilon, v}(r, s)=0\right\} & =\left\{(r, s) \in \mathbb{R}^{2} \quad \mid r+s=\varepsilon^{n-2}-L_{v}(r, s)-Q_{v}(r, s)\right\} \\
& \subseteq\left\{(r, s) \in \mathbb{R}^{2} \mid(1-c) \varepsilon^{n-2} \leq r+s \leq(1+c) \varepsilon^{n-2}\right\} \\
& =: Z_{\varepsilon}
\end{aligned}
$$

If we set $r_{0}:=\varepsilon^{n-2} / 2$ for every $v \in B_{\gamma}\left(r_{\varepsilon}\right)$, there must exist a real number $s_{0}(v)$ such that $\left(r_{0}, s_{0}(v)\right) \in Z_{\varepsilon}$ and $G_{\varepsilon, v}\left(r_{0}, s_{0}(v)\right)=0$.

Obviously the functions $G_{\varepsilon, v}$ depend smoothly on variables $r$ and $s$ and it is not hard to show that their partial derivatives at the origin are uniformly bounded with respect to $v \in B_{\gamma}\left(r_{\varepsilon}\right)$. We can also provide the partial derivatives $\partial_{r} \cdot G_{\varepsilon, v}(0,0)$ with a uniform lower bound. In fact, for sufficiently small $\varepsilon$, we can compute

$$
\begin{aligned}
\left|\partial_{r} \cdot G_{\varepsilon, v_{j}}(0,0)\right| & =\left|\int_{M_{1}} K_{1} \cdot\left(1+v_{j}\right) \mathrm{dvol}_{g_{1}}\right| \\
& \geq\left|\int_{M_{1}} K_{1} \mathrm{dvol}_{g_{1}}\right|-\int_{M_{1}}\left|K_{1}\right|\left|v_{j}\right| \mathrm{dvol}_{g_{1}}
\end{aligned}
$$




$$
\begin{aligned}
& \geq\left|\int_{M_{1}} K_{1} \mathrm{dvol}_{g_{1}}\right|-\left\|v_{j}\right\|_{\mathcal{C}^{0}(M)} \int_{M_{1}}\left|K_{1}\right| \mathrm{dvol}_{g_{1}} \\
& \geq \frac{1}{2}\left|\int_{M_{1}} K_{1} \mathrm{dvol}_{g_{1}}\right|
\end{aligned}
$$

Observe that the bound does not depend on $v$ and that the same is true for $\partial_{s} \cdot G_{\varepsilon, v}$.

Arguing by contradiction and using these estimates it is possible to deduce that there exists a positive constant $C>0$ and a positive real number $R>0$ such that both the first partial derivatives $\left|\partial_{r} \cdot G_{\varepsilon, v}\right|$ and $\left|\partial_{s} \cdot G_{\varepsilon, v}\right|$ are greater than $C$ in $B_{R}(0,0)$, for every $v \in B_{\gamma}\left(r_{\varepsilon}\right)$.

Provided $\varepsilon$ is sufficiently small, we see that the set $Z_{\varepsilon} \cap\{r, s \geq 0\}$ lies in the ball of radius $R$ centered at the origin, hence it is possible to apply the implicit function Theorem to the functions $G_{\varepsilon, v}$ around the points $\left(r_{0}, s_{0}(v)\right)$. As a consequence, we obtain, for every $v \in B_{\gamma}\left(r_{\varepsilon}\right)$, an open neighborhood $U(v)$ of $r_{0}$, an open neighborhood $V(v)$ of $s_{0}(v)$ and a smooth function $f_{v}: U(v) \longrightarrow V(v)$ such that $G_{\varepsilon, v}\left(r, f_{v}(r)\right)=0$ for every $r \in U(v)$.

Since it is possible to extend each implicit function $f_{v}$ to the interval $(0,(1-$ c) $\varepsilon^{n-2}$ ), we can suppose that there exists an open neighborhood $U$ of $r_{0}$ and an open neighborhood $V$ of every $s_{0}(v)$ such that it is possible to choose $U(v)=U$ and $V(v)=V$ for every $v \in B_{\gamma}\left(r_{\varepsilon}\right)$.

Let us fucus now on the family of functions $\left\{f_{v}\right\}_{v \in B_{\gamma}\left(r_{\varepsilon}\right)}$. Since each $f_{v}$ is a uniformly continuous function, we can extend all of them to the compact set $\bar{U}$. This way we have obtained a family of functions $f_{v}: \bar{U} \longrightarrow \bar{V}$ defined on the same compact set $\bar{U}$ and all bounded by the same constant, namely $(1+c) \varepsilon^{n-2}$.

Our aim is now to show that the $f_{v}$ 's admit the same Lipschitz's constant. First remember that the $f_{v}$ 's satisfy

$$
f_{v}(r)=\int_{M} S_{g_{\varepsilon}} \operatorname{dvol}_{g_{\varepsilon}}-r+L_{v}\left(r, f_{v}(r)\right)+Q_{v}\left(r, f_{v}(r)\right)
$$

As a consequence, for $r, r^{\prime} \in \bar{U}$ and suitable positive constants $C_{1}$ and $C_{2}$, we have

$$
\begin{aligned}
\left|f_{j}(r)-f_{j}\left(r^{\prime}\right)\right| \leq & \left|r-r^{\prime}\right|+\left|L_{v}\left(r, f_{v}(r)\right)-L_{v}\left(r^{\prime}, f_{v}\left(r^{\prime}\right)\right)\right| \\
& +\left|Q_{v}\left(r, f_{v}(r)\right)-Q_{v}\left(r^{\prime}, f_{v}\left(r^{\prime}\right)\right)\right| \\
\leq & \left|r-r^{\prime}\right|+\int_{M_{1}}\left|K_{1}\right| \mathrm{dvol}_{g_{1}} \cdot\|v\|_{\mathcal{C}^{0}(M)} \cdot\left|r-r^{\prime}\right| \\
& +\int_{M_{2}}\left|K_{2}\right| \operatorname{dvol}_{g_{2}} \cdot\|v\|_{\mathcal{C}^{0}(M)} \cdot\left|f_{v}(r)-f_{v}\left(r^{\prime}\right)\right| \\
& +C_{1} \varepsilon^{n-2} \cdot\left|r-r^{\prime}\right|+C_{2} \varepsilon^{n-2} \cdot\left|f_{j}(r)-f_{j}\left(r^{\prime}\right)\right|
\end{aligned}
$$


It follows that for small enough $\varepsilon$

$$
\begin{aligned}
\left|f_{j}(r)-f_{j}\left(r^{\prime}\right)\right| & \leq \frac{1+\|v\|_{\mathcal{C}^{0}(M)} \int_{M_{1}}\left|K_{1}\right| \mathrm{dvol}_{g_{1}}+C_{1} \varepsilon^{n-2}}{1-\|v\|_{\mathcal{C}^{0}(M)} \int_{M_{2}}\left|K_{2}\right| \mathrm{dvol}_{g_{2}}+C_{2} \varepsilon^{n-2}} \cdot\left|r-r^{\prime}\right| \\
& \leq 4 \cdot\left|r-r^{\prime}\right|
\end{aligned}
$$

Thanks to the Ascoli-Arzelà Theorem, any sequence of functions contained in the family $\left\{f_{v}\right\}_{v \in B_{\gamma}\left(r_{\varepsilon}\right)}$ converges (up to a subsequence) to a continuous function $f$ with respect to the norm $\|\cdot\|_{\mathcal{C}^{0}(\bar{U})}$. Moreover $f$ has the same bound and the same Lipschitz's constant as the $f_{v}$ 's. In the following we will use the continuity of $f$ to kill the rough projection of the error term in the Eq. (58).

We summarize the results obtained so far in this section: for all functions $v \in$ $B_{\gamma}\left(r_{\varepsilon}\right)$ we have found a smooth function $f_{v}$ defined on a neighborhood $\bar{U}$ of $r_{0}=\varepsilon^{n-2} / 2$ such that the condition

$$
\int_{M} \tilde{F}_{\varepsilon}\left(v, r, f_{v}(r)\right) \operatorname{dvol}_{\tilde{g}_{\varepsilon}}=0
$$

is verified for all $r \in \bar{U}$.

Having the orthogonality condition, we can define the operator $\tilde{P}_{\varepsilon}$ as in Sect. 4, namely

$$
\tilde{P}_{\varepsilon}:=\tilde{G}_{\varepsilon} \circ \tilde{F}_{\varepsilon}
$$

For such an operator it is easy to obtain (modulo obvious modifications) the analog of Lemma 2, with the same definition of the radius $r_{\varepsilon}$.

It is also immediate to prove that for sufficiently small $\varepsilon>0, \tilde{P}_{\varepsilon}$ is a contraction mapping and more precisely

$$
\left\|\tilde{P}_{\varepsilon}(u)-\tilde{P}_{\varepsilon}(v)\right\|_{\mathcal{C}_{\gamma}^{0}(M)} \leq D \varepsilon \cdot\|u-v\|_{\mathcal{C}_{\gamma}^{0}(M)}
$$

for a suitable constant $D>0$. In particular the sequence defined by $v_{j}:=\tilde{P}_{\varepsilon}^{j}(0)$ converges with respect to the norm $\|\cdot\|_{\mathcal{C}_{\gamma}^{0}(M)}$ to a function $v_{\varepsilon} \in B_{\gamma}\left(r_{\varepsilon}\right)$. Also notice that at the same time (up to consider a subsequence of the $v_{j}$ 's) the sequence of functions $f_{v_{j}}$ converges as well to a continuous function $f$. Hence, for every $r \in \bar{U}$, $v_{\varepsilon}$ verifies the identity

$$
\Delta_{\tilde{g}_{\varepsilon}} v_{\varepsilon}=\tilde{F}_{\varepsilon}\left(v_{\varepsilon}, r, f(r)\right)-\lambda_{\tilde{F}_{\varepsilon}\left(v_{\varepsilon}, r, f(r)\right)} \beta_{\varepsilon}
$$

We are now ready to discuss the sign of the term $\lambda_{\tilde{F}_{\varepsilon}\left(v_{\varepsilon}, r, f(r)\right)}$ which appears in this formula.

As in the general case, it turns out that the sign of the rough projection is determined by the sign of

$$
\begin{aligned}
\lambda_{\tilde{F}_{\varepsilon}\left(v_{\varepsilon}, r, f(r)\right)}^{(0)}= & \frac{1}{\int_{M} \chi_{1}+\chi_{2} \operatorname{dvol}_{\tilde{g}_{\varepsilon}}} \int_{M} \tilde{F}_{\varepsilon}\left(v_{\varepsilon}\right)\left(\chi_{1}-\chi_{2}\right) \operatorname{dvol} \tilde{g}_{\varepsilon} \\
& +\frac{1}{\int_{M} \chi_{1}+\chi_{2} \operatorname{dvol}_{\tilde{g}_{\varepsilon}}} \int_{M} \Delta_{g_{\varepsilon}}\left(\chi_{1} \tilde{u}_{P}^{\varepsilon}\right)-\Delta_{g_{\varepsilon}}\left(\chi_{2} \tilde{u}_{P}^{\varepsilon}\right) \operatorname{dvol} \tilde{g}_{\varepsilon}
\end{aligned}
$$


where $\tilde{F}_{\varepsilon}\left(v_{\varepsilon}\right)=-c_{m} \cdot\left(S_{g_{1}+r h_{1}}+S_{g_{\varepsilon}}+S_{g_{2}+f(r) h_{2}}\right) \cdot\left(1+v_{\varepsilon}\right)$ and $\tilde{u}_{P}^{\varepsilon}$ is the same as in Sect. 5.

As in the previous section we have

$$
\begin{aligned}
& \int_{M} \Delta_{g_{\varepsilon}}\left(\chi_{1} \tilde{u}_{P}^{\varepsilon}\right) \quad \operatorname{dvol}_{\tilde{g}_{\varepsilon}}=\mathcal{O}\left(\mathrm{e}^{-\alpha_{1}} \varepsilon^{n-2}\right) \\
& \int_{M} \Delta_{g_{\varepsilon}}\left(\chi_{i} \tilde{u}_{P}^{\varepsilon}\right) \operatorname{dvol}_{\tilde{g}_{\varepsilon}}=\mathcal{O}\left(\mathrm{e}^{-\alpha_{2}} \varepsilon^{n-2}\right)
\end{aligned}
$$

Concerning the other summand, we compute

$$
\begin{aligned}
-\frac{1}{c_{m}} \int_{M} \tilde{F}_{\varepsilon}\left(v_{\varepsilon}\right)\left(\chi_{1}-\chi_{2}\right) \operatorname{dvol}_{\tilde{g}_{\varepsilon}}= & r \int_{M_{1}} K_{1} \mathrm{dvol}_{g_{1}}-f(r) \int_{M_{2}} K_{2} \mathrm{dvol}_{g_{2}} \\
& +\int_{M} S_{g_{\varepsilon}}\left(\chi_{1}-\chi_{2}\right) \operatorname{dvol}_{\tilde{g}_{\varepsilon}}+\mathcal{O}\left(\varepsilon^{n-2+\gamma}\right) \\
= & r-f(r)+\mathcal{O}\left(\mathrm{e}^{-\alpha_{1}} \varepsilon^{n-2}\right)+\mathcal{O}\left(\mathrm{e}^{-\alpha_{2}} \varepsilon^{n-2}\right) \\
& +\mathcal{O}\left(\varepsilon^{n-2+\gamma}\right)
\end{aligned}
$$

Hence it is clear that for small $\varepsilon$ and large enough $\alpha_{1}$ and $\alpha_{2}$, the sign of the rough projection is determined by the term $r-f(r)$.

Since it is always possible to choose $r$ either in a region where $f(r)>r$ or in a region where $f(r)<r$ and $f$ is a continuous function, we conclude that there exist $\bar{r} \in \bar{U}$ such that

$$
\lambda_{\tilde{F}_{\varepsilon}\left(v_{\varepsilon}, \bar{r}, f(\bar{r})\right)}=0
$$

This completes the proof of Theorem 2 .

To conclude this section, we would like to make some comment about the non Ricci flat hypothesis. Following [10,11], the compact Riemannian manifolds without boundary can be divided in the following three classes:

$\left(1_{+}\right)$Manifolds admitting a Riemannian metric whose scalar curvature is nonnegative and not identically zero.

$\left(1_{0}\right)$ Manifolds admitting a Riemannian metric with non-negative scalar curvature, but not in class $\left(1_{+}\right)$.

(1_) Manifolds not in classes $\left(1_{+}\right)$or $\left(1_{0}\right)$.

This classification is justified by the following classical result

Theorem 6. (Trichotomy Theorem, $[10,11])$ Let $M$ be a compact connected Riemannian manifold without boundary, of dimension $\geq 3$, then we have

1. If $M$ belongs to class $\left(1_{+}\right)$, every smooth function is realized as the scalar curvature of some Riemannian metric on $M$. 
2. If $M$ belongs to class $\left(1_{0}\right)$, then a function $S \in \mathcal{C}^{\infty}(M)$ is the scalar curvature of some Riemannian metric on $M$ if and only if either $S(p)<0$ for some point $p \in M$, or else $S \equiv 0$. Moreover, if the scalar curvature of some metric $g$ vanishes identically, then $g$ is Ricci flat.

3. If $M$ belongs to class (1-), then $S \in \mathcal{C}^{\infty}(M)$ is the scalar curvature of some metric if and only if $S(p)<0$ for some point $p \in M$.

In our situation, the initial manifolds $M_{1}$ and $M_{2}$ carry a scalar flat metric, then a priori they might belong to class $\left(1_{+}\right)$or to class $\left(1_{0}\right)$. Because of the Trichotomy Theorem the non Ricci flat hypothesis implies that they must belong to class $\left(1_{+}\right)$. As it is stated in the Theorem above, the manifolds which are in the class $\left(1_{+}\right)$ are the ones for which the prescribed scalar curvature problem has no obstructions. This yields a philosophical justification of the non Ricci flat hypothesis. In other word, if we want to construct a scalar flat metric on the generalized connected sum, we need (so far) to handle manifolds which are very flexible concerning the scalar curvature.

\section{Generalized gluing for time symmetric initial data}

In this section we will discuss an interesting physical application of Theorem 2. To fix the setting, let $(Z, \gamma)$ be an $(m+1)$-dimensional Lorentzian manifold. The hyperbolic Einstein system for the vacuum spacetime is given by

$$
\operatorname{Ric}_{\gamma}=0
$$

In the early 1950s Y. Choquet-Bruhat showed in a famous paper [4] that a solution to this system can be obtained from a suitable choice of initial data sets. An initial data set for the Einstein problem consists of an $m$-dimensional space-like hypersurface $M \subset Z$ and two symmetric tensors $g$ and $\Pi$ (which correspond to the induced metric and to the second fundamental form of $M$, respectively) verifying the following system, also known as the Einstein constraint equations

$$
\begin{array}{r}
\operatorname{div}_{g} \Pi-d\left(\operatorname{tr}_{g} \Pi\right)=0 \\
S_{g}-|\Pi|_{g}^{2}+\left(\operatorname{tr}_{g} \Pi\right)^{2}=0
\end{array}
$$

where $S_{g}$ indicates the (intrinsic) scalar curvature of the Riemannian manifold $(M, g)$.

A natural idea is to produce new solutions to (69) by gluing together two suitable initial data (or Cauchy data) sets. This has been done in the case of the connected sum at points of two constant mean curvature (briefly CMC) solutions to the constraints, with second fundamental form $\Pi$ nonidentically zero (see [8]).

For physical reason, when the second fundamental form $\Pi$ is identically equal to zero, the Cauchy data set $(M, g, \Pi)$ is said to be time symmetric (roughly, a time symmetric slice). In this case, it is immediate to check that the system (70)-(71) simply reduces to

$$
S_{g}=0
$$


Hence the (generalized) gluing of two time symmetric initial data set $\left(M_{1}, g_{1}, \Pi_{1}\right)$ and $\left(M_{2}, g_{2}, \Pi_{2}\right)$ simply reduces as well to the construction of a scalar flat metric on the (generalized) connected sum of two scalar flat Riemannian manifolds. In fact the second fundamental form on the generalized connected sum can be defined to be identically zero and this trivially yields a gluing when both $\Pi_{1}$ and $\Pi_{2}$ are identically zero.

If we consider two time symmetric initial data which are non Ricci flat, then Theorem 2 yields a (generalized) gluing construction for such Cauchy data sets. Hence we can state the following

Corollary 7. Let $\left(M_{1}, g_{1}, \Pi_{1}\right)$ and $\left(M_{2}, g_{2}, \Pi_{2}\right)$ be two m-dimensional non Ricci flat solutions to the system (70)-(71) with $\Pi_{1} \equiv 0 \equiv \Pi_{2}$ and let $\left(K, g_{K}\right)$ be a common isometrically embedded Riemannian submanifold whose dimension $k$ is such that $n:=m-k \geq 3$. Moreover suppose that the normal bundles of $K$ in $M_{1}$ and $M_{2}$ are diffeomorphic, then there exists a real number $\varepsilon_{0}$ such that, for every $\varepsilon \in\left(0, \varepsilon_{0}\right)$, the generalized connected sum $M_{\varepsilon}=M_{1} \sharp_{K, \varepsilon} M_{2}$ of $M_{1}$ and $M_{2}$ along $K$, obtained by excising a tubular neighborhood of $K$ of size $\varepsilon$ from both the initial manifolds and identifying the left over boundaries, can be endowed with a new metric $\bar{g}_{\varepsilon}$ and a new second fundamental forms $\bar{\Pi}_{\varepsilon} \equiv 0$ such that $\left(M_{\varepsilon}, \bar{g}_{\varepsilon}, \bar{\Pi}_{\varepsilon}\right)$ is still a solution of the Einstein constraints (70)-(71). Moreover the new metrics tend to the old ones on the compact sets of $M_{i} \backslash K, i=1,2$ in the $\mathcal{C}^{2}$ topology, as $\varepsilon \rightarrow 0$.

Open Access This article is distributed under the terms of the Creative Commons Attribution Noncommercial License which permits any noncommercial use, distribution, and reproduction in any medium, provided the original author(s) and source are credited.

\section{References}

[1] Anderson, M.: Dehn filling and Einstein metrics in higher dimensions, arxiv:math.DG/ 0303260 (2003)

[2] Aubin, T.: Some nonlinear Problems in Riemannian Geometry. Springer Monographs in Mathematics. Springer, Berlin (1998)

[3] Besse, A.L.: Einstein Manifolds. Spinger, Berlin (1987)

[4] Choquet-Bruhat, Y.: Théorème d'existence pour certains systèmes d'équations aux dérivées partielles non linéaires. Acta Math. 88, 141-225 (1952)

[5] Gilbarg, D., Trudinger, N.: Elliptic Partial Differential Equation of Second Order. Springer, Berlin (1983)

[6] Gromov, M., Lawson, H.B.: The classification of simply connected manifolds of positive scalar curvature. Ann. Math. 111, 423-434 (1980)

[7] Isenberg, J., Maxwell, D., Pollack, D.: A gluing construction for non vacuum solutions of the Einstein constraint equations. Adv. Theor. Phys. 9, 129-172 (2005)

[8] Isenberg, J., Mazzeo, R., Pollack, D.: Gluing and wormholes for the Einstein constraint equations. Comm. Math. Phys. 231, 529-568 (2002)

[9] Joyce, D.: Constant scalar curvature metrics on connected sums. Int. J. Math. Math. Sci. 7, 405-450 (2003)

[10] Kazdan, J.L., Warner, F.: Existence and conformal deformation of metrics with prescribed Gaussian and scalar curvature. Ann. Math. 101, 317-331 (1975) 
[11] Kazdan, J.L., Warner, F.: Scalar curvature and conformal deformation of Riemannian structure. J. Differ. Geom. 10, 113-134 (1975)

[12] Mazzeo, R., Pacard, F.: Constant scalar curvature metrics with isolated singularities. Duke Math. J. 99, 353-418 (1999)

[13] Mazzeo, R., Pacard, F.: Constant mean curvature surfaces with Delaunay ends. Comm. Anal. Geom. 9, 169-237 (2001)

[14] Mazzeo, R., Pacard, F., Pollack, D.: Connected sums of constant mean curvature surfaces in Euclidean 3 space. J. Reine Angew. Math. 536, 115-165 (2001)

[15] Mazzeo, R., Pollack, D., Uhlenbeck, K.: Connected sums constructions for constant scalar curvature metrics. Topol. Method Nonlinear Anal. 6, 207-233 (1995)

[16] Mazzieri, L.: Generalized connected sum construction for nonzero constant scalar curvature metrics. Comm. Part. Differ. Equ. 33, 1-17 (2008)

[17] Schoen, R., Yau, S.T.: On the structure of manifolds with positive scalar curvature. Manuscr. Math. 28, 159-183 (1979) 